\title{
Subharmonic transitions and Bloch-Siegert shift in electrically driven spin resonance
}

\author{
Judit Romhányi, ${ }^{1,2}$ Guido Burkard, ${ }^{3}$ and András Pályi ${ }^{2,4}$ \\ ${ }^{1}$ Leibniz Institute for Solid State and Materials Research, IFW-Dresden, D-01171 Dresden, Germany \\ ${ }^{2}$ Institute of Physics, Eötvös University, Budapest, Hungary \\ ${ }^{3}$ Department of Physics, University of Konstanz, D-78457 Konstanz, Germany \\ ${ }^{4}$ MTA-BME Condensed Matter Research Group, Budapest University of Technology and Economics, Budapest, Hungary
}

(Received 23 April 2015; revised manuscript received 14 July 2015; published 11 August 2015)

\begin{abstract}
We theoretically study coherent subharmonic (multiphoton) transitions of a harmonically driven spin. We consider two cases: magnetic resonance (MR) with a misaligned, i.e., nontransversal, driving field, and electrically driven spin resonance (EDSR) of an electron confined in a one-dimensional, parabolic quantum dot, subject to Rashba spin-orbit interaction. In the EDSR case, we focus on the limit where the orbital level spacing of the quantum dot is the greatest energy scale. Then, we apply time-dependent Schrieffer-Wolff perturbation theory to derive a time-dependent effective two-level Hamiltonian, allowing us to describe both MR and EDSR using the Floquet theory of periodically driven two-level systems. In particular, we characterize the fundamental (singlephoton) and the half-harmonic (two-photon) spin transitions. We demonstrate the appearance of two-photon Rabi oscillations, and analytically calculate the fundamental and half-harmonic resonance frequencies and the corresponding Rabi frequencies. For EDSR, we find that both the fundamental and the half-harmonic resonance frequencies change upon increasing the strength of the driving electric field, which is an effect analogous to the Bloch-Siegert shift known from MR. Remarkably, the drive-strength-dependent correction to the fundamental EDSR resonance frequency has an anomalous, negative sign, in contrast to the corresponding Bloch-Siegert shift in MR which is always positive. Our analytical results are supported by numerical simulations, as well as by qualitative interpretations for simple limiting cases.
\end{abstract}

DOI: 10.1103/PhysRevB.92.054422

PACS number(s): 71.70.Ej, 73.21.La, 76.20.+q

\section{INTRODUCTION}

Magnetic resonance (MR) is an established method to coherently control the quantum state of spins. A simple example is a spin- $1 / 2$ electron subject to a time-dependent magnetic field [1-4], described by the Hamiltonian

$$
H=-\frac{1}{2} g \mu_{B} \mathbf{B}(t) \cdot \boldsymbol{\sigma},
$$

where the magnetic field $\mathbf{B}(t)=\left(0,-B_{\mathrm{ac}} \cos \omega t, B\right)$ consists of a "longitudinal" static component $B$ and a "transverse" ac component characterized by the drive strength $B_{\mathrm{ac}}$ and the drive frequency $\omega$, and couples to the electron spin represented by the vector $\boldsymbol{\sigma}=\left(\sigma_{x}, \sigma_{y}, \sigma_{z}\right)$ of Pauli matrices.

A typical initial-value problem considered in MR is when the initial state of the spin $\psi(t=0)=|\uparrow\rangle$ is the ground state of the static Hamiltonian, $-\frac{1}{2} g \mu_{B} B \sigma_{z}$, and driving is switched on abruptly at $t=0$. In the case of weak driving $B_{\mathrm{ac}} \ll B$, the rotating wave approximation (RWA) often provides a satisfactory description of the dynamics. Using this approximation, one finds the following simple phenomenology. If the resonance condition $\hbar \omega=g \mu_{B} B$ is fulfilled, the drive will induce complete Rabi oscillations resulting in a transition probability $P_{\downarrow}(t) \equiv|\langle\downarrow \mid \psi(t)\rangle|^{2}=$ $\sin ^{2}(\Omega t / 2)$, where $\Omega=g \mu_{B} B_{\text {ac }} /(2 \hbar)$ is called the Rabi frequency. Otherwise, i.e., in the case of a finite detuning $\delta=$ $\omega-g \mu_{B} B / \hbar$ between the drive frequency and the resonance frequency, one finds incomplete Rabi oscillations with a $\delta$ dependent frequency: $P_{\downarrow}(t)=P_{\downarrow}^{\max } \sin ^{2}\left(\sqrt{\delta^{2}+\Omega^{2}} t / 2\right)$, with $P_{\downarrow}^{\max }=\Omega^{2} /\left(\Omega^{2}+\delta^{2}\right)<1$.

Still focusing on the weak-driving regime $B_{\mathrm{ac}} \ll B$, one can go beyond the RWA, e.g., by numerical simulations or analytical techniques such as the Floquet perturbation theory
[2]. Then, a richer phenomenology is revealed, including (i) subharmonic or "multiphoton" resonances [2], (ii) drivestrength-dependent Bloch-Siegert shifts [1] (BSSs) of the resonance frequencies, and (iii) Bloch-Siegert oscillations modulating the simple Rabi oscillations [1]. We restrict our attention to (i) and (ii) here.

(i) In the case of a transverse ac field, such as the example used in Eq. (1), odd subharmonic resonances appear [2]. Rabi oscillations are obtained not only for the fundamental resonance $\omega \approx g \mu_{B} B / \hbar$, but also when $\omega \approx g \mu_{B} B /(N \hbar)$ with $N=3,5,7, \ldots$ In the case of a misaligned, nontransversal, ac field, such as $\mathbf{B}(t)=\left(0,-B_{\mathrm{ac}} \cos \theta \cos \omega t\right.$, $\left.B-B_{\mathrm{ac}} \sin \theta \cos \omega t\right)$ with $0<\theta<\pi / 2$, both even and odd subharmonics are present. The Rabi frequency $\Omega_{\text {res }}^{(N)}$ at the $N$-photon subharmonic resonance is weaker than that of the fundamental one: $\Omega_{\mathrm{res}}^{(N)} \propto B_{\mathrm{ac}}^{N} / B^{(N-1)}$.

(ii) The resonance frequencies $\omega_{\text {res }}^{(N)}$ (i.e., the drive frequencies where complete Rabi oscillations are induced) increase with increasing drive strength, by an amount that depends on $N$, and is proportional to $B_{\mathrm{ac}}^{2} / B$.

In many situations, it is be more convenient to control spins using an ac electric field rather than an ac magnetic field. For example, if an electron spin is electrostatically confined in a quantum dot (QD), then an ac electric field can be easily created by applying an ac voltage component of the confinement gate electrodes. Along these lines, electrically driven spin resonance [5-11] (EDSR) of individual electron spins was demonstrated in a variety of materials [12-23]. As the ac electric field couples to the orbital degree of freedom of the electron and has no direct effect on the spin, a sufficiently strong coupling mechanism between the orbit and spin is required for EDSR. Such a coupling can be supplied by spin-orbit 

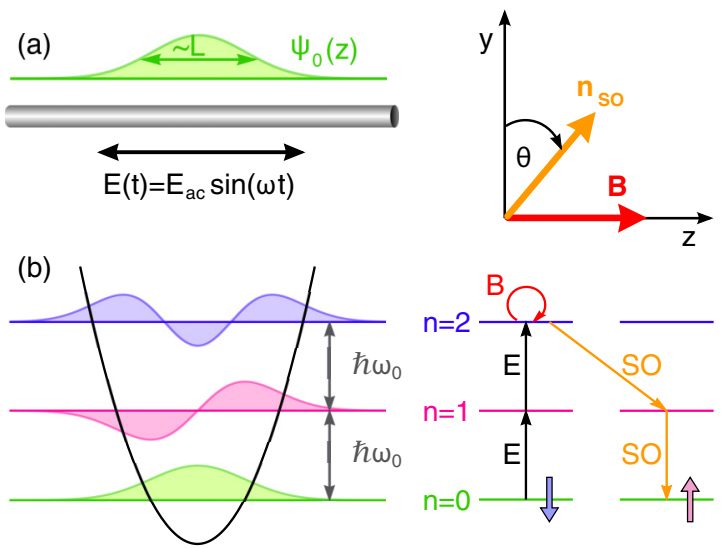

FIG. 1. (Color online) Electrically driven spin resonance in a 1D quantum dot. (a) An electron occupying the ground state $\psi_{0}(z)$ of a parabolic confinement potential is excited by an ac electric field of amplitude $E_{\mathrm{ac}}$ and frequency $\omega$. The electron is subject to a homogeneous magnetic field $\mathbf{B}$ and spin-orbit interaction characterized by the direction $\mathbf{n}_{\text {SO }}$. (b) Left: Orbital levels labeled by the oscillator quantum number $n$, separated by the level spacing $\hbar \omega_{0}$. Right: Diagram representing one of the many fifth-order virtual processes contributing to the half-harmonic resonance. Horizontal lines represent the energy eigenstates of the harmonic oscillator Hamiltonian. Arrows labeled by $E, B$, and SO correspond to matrix elements of the ac electric field, magnetic field, and spin-orbit Hamiltonians, respectively. Note that the spin-orbit Hamiltonian provides both spin-flip and spin-conserving matrix elements only if both $\cos \theta$ and $\sin \theta$ are nonzero. [See Eq. (36).]

interaction, hyperfine interaction, or an inhomogeneous magnetic field.

Recent experimental [20,23-26] and theoretical [27-34] studies addressed subharmonic resonances in EDSR. One mechanism that leads to subharmonic resonances in EDSR is the appearance of higher harmonics $N \omega$ of the drive frequency $\omega$ in the induced orbital dynamics [29,31]. In this case, the time-dependent effective magnetic fields caused by the orbital dynamics will also have components at frequency $N \omega$, leading to Rabi oscillations as $N \omega$ matches the Zeeman splitting. Higher harmonics in the orbital dynamics arise naturally if the confinement potential is anharmonic, or if the driving electric field is inhomogeneous. Subharmonic EDSR resonances can also arise in the presence of harmonic confinement and homogeneous ac electric field, if the gradient of the effective magnetic fields is inhomogeneous; this is the case, e.g., if the the effective magnetic field is spatially localized or disordered [32]. A third mechanism, able to cause strong subharmonics with large $N$, is provided by Landau-Zener dynamics in the vicinity of level anticrossings [26,34].

In this work, we theoretically describe the characteristics of the half-harmonic resonance in MR with a misaligned ac field, as well as in EDSR. First, we use Floquet perturbation theory [2] to characterize the parameter dependence of the half-harmonic resonance frequency and the corresponding Rabi frequency in the case of MR; in particular, the BSS is calculated. As for EDSR, we study a model [35] (see Fig. 1) in which a single electron is parabolically confined in a one-dimensional (1D) quantum dot, and is subject to a dc magnetic field, an ac electric field, and spin-orbit interaction of Rashba type, the latter three being spatially homogeneous. We show that the half-harmonic resonance does arise in this model, despite the harmonic confinement and homogeneous ac electric field. In the perturbative regime of this model, i.e., when the orbital level spacing $\hbar \omega_{0}$ dominates over other energy scales, we analytically derive the parameter dependence of the half-harmonic resonance frequency and the corresponding Rabi frequency. This is achieved via a combination of time-dependent Schrieffer-Wolff perturbation theory (TDSW), which is used to obtain a $2 \times 2$ effective "two-level" or "qubit" Hamiltonian $\tilde{\mathcal{H}}_{q}$, and Floquet perturbation theory, applied to describe the qubit dynamics governed by $\tilde{\mathcal{H}}_{q}$.

This paper is structured as follows. In Sec. II we summarize our main results. Sections III and IV are dedicated to the detailed discussion of MR and EDSR, respectively. In them we formulate the problems, derive analytical solutions, and compare these with numerical simulations where called for. In Sec. V we give a conclusion of our findings.

\section{SUMMARY OF THE RESULTS}

In this section, we summarize the main results that are derived in later sections. Let us start with the case of spin-1/2 MR with a misaligned ac field. The parameters of the model are $\tilde{B}$, the energy scale of the static magnetic field along the $z$ axis; $\tilde{B}_{\text {ac }}$, the energy scale of the ac magnetic field oriented in the $y z$ plane; and $\theta$, the angle enclosed by the ac field and the $y$ axis. (For more details, see Sec. III.) The fundamental (or single-photon) resonance frequency $\omega_{\text {res }}^{(1)}$, that is, the drive frequency at which the Rabi oscillations are complete, deviates from the Zeeman splitting:

$$
\hbar \omega_{\mathrm{res}}^{(1)}=\tilde{B}+\hbar \omega_{\mathrm{BSS}}^{(1)},
$$

where the second term on the right-hand side is the BSS and has the form

$$
\hbar \omega_{\mathrm{BSS}}^{(1)}=\frac{\tilde{B}_{\mathrm{ac}}^{2} \cos ^{2} \theta}{16 \tilde{B}} .
$$

The Rabi frequency at the fundamental resonance is

$$
\hbar \Omega_{\mathrm{res}}^{(1)}=\frac{\tilde{B}_{\mathrm{ac}}}{2} \cos \theta .
$$

Similarly to the fundamental resonance, the half-harmonic (two-photon) resonance also acquires a positive BSS:

$$
\hbar \omega_{\mathrm{res}}^{(2)}=\frac{\tilde{B}}{2}+\hbar \omega_{\mathrm{BSS}}^{(2)},
$$

where

$$
\hbar \omega_{\mathrm{BSS}}^{(2)}=\frac{\tilde{B}_{\mathrm{ac}}^{2} \cos ^{2} \theta}{6 \tilde{B}} .
$$

The Rabi frequency at the half-harmonic resonance is

$$
\hbar \Omega_{\mathrm{res}}^{(2)}=\frac{\tilde{B}_{\mathrm{ac}}^{2} \sin 2 \theta}{4 \tilde{B}} .
$$

Note that the resonance frequencies above are expressed up to second order in the small energy scale $\tilde{B}_{\text {ac }} \ll \tilde{B}$. For a detailed discussion of these results, see Sec. III D. 
In the case of EDSR in a 1D parabolic QD, the parameters characterizing the model are as follows. The orbital level spacing $\hbar \omega_{0}$ is the dominant energy scale; the static magnetic field, oriented along the $z$ axis, is characterized by the Zeemansplitting energy scale $\tilde{B}$; spin-orbit interaction is described by the energy scale $\tilde{\alpha}$ and the unit vector $\mathbf{n}_{\text {so }}=(0, \cos \theta, \sin \theta)$ which points along the spin-orbit field; and the energy scale $\tilde{E}_{\text {ac }}$ describing the strength of the driving ac electric field. We find the following results for the fundamental resonance frequency:

$$
\hbar \omega_{\mathrm{res}}^{(1)}=\tilde{B}+\hbar \omega_{g}^{(1)}+\hbar \omega_{\mathrm{nlZ}}^{(1)}+\hbar \omega_{\mathrm{BSS}}^{(1)},
$$

where the correction consists of a $g$-factor renormalization term,

$$
\hbar \omega_{g}^{(1)}=-\frac{2 \tilde{B} \tilde{\alpha}^{2} \cos ^{2} \theta}{\hbar^{2} \omega_{0}^{2}}\left(1-\frac{\tilde{\alpha}^{2}\left(1+\sin ^{2} \theta\right)}{\hbar^{2} \omega_{0}^{2}}\right),
$$

a term describing the nonlinear Zeeman effect,

$$
\hbar \omega_{\mathrm{nlZ}}^{(1)}=\frac{2 \tilde{B}^{3} \tilde{\alpha}^{2} \cos ^{2} \theta}{\hbar^{4} \omega_{0}^{4}}
$$

and a correction that is second order in the drive strength $\tilde{E}_{\mathrm{ac}}$, hence analogous to the BSS:

$$
\hbar \omega_{\mathrm{BSS}}^{(1)}=-\frac{\tilde{B} \tilde{\alpha}^{2} \tilde{E}_{\mathrm{ac}}^{2} \cos ^{2} \theta}{\hbar^{4} \omega_{0}^{4}} .
$$

Note that the sign of this BSS is negative, in contrast to the positive sign in the case of MR [Eqs. (3) and (6)]; an interpretation of this anomalous sign is given in Sec. V, paragraph (4). The Rabi frequency at the fundamental resonance is

$$
\hbar \Omega_{\mathrm{res}}^{(1)}=2 \frac{\tilde{B} \tilde{E}_{\mathrm{ac}} \tilde{\alpha} \cos \theta}{\hbar^{2} \omega_{0}^{2}}\left(1+\frac{\tilde{B}^{2}-2 \tilde{\alpha}^{2}}{\hbar^{2} \omega_{0}^{2}}\right) .
$$

The half-harmonic resonance frequency is shifted with respect to the half of the fundamental resonance frequency:

$$
\hbar \omega_{\mathrm{res}}^{(2)}=\frac{1}{2}\left(\tilde{B}+\hbar \omega_{g}^{(1)}+\hbar \omega_{\mathrm{nlZ}}^{(1)}\right)+\hbar \omega_{\mathrm{BSS}}^{(2)},
$$

where the drive-strength-dependent BSS is expressed as

$$
\hbar \omega_{\mathrm{BSS}}^{(2)}=\frac{2 \tilde{B} \tilde{\alpha}^{2} \tilde{E}_{\mathrm{ac}}^{2} \cos ^{2} \theta}{3 \hbar^{4} \omega_{0}^{4}} .
$$

The Rabi frequency at the half-harmonic resonance is

$$
\hbar \Omega_{\mathrm{res}}^{(2)}=\frac{\tilde{B} \tilde{\alpha}^{2} \tilde{E}_{\mathrm{ac}}^{2} \sin (2 \theta)}{\left(\hbar \omega_{0}\right)^{4}} .
$$

Note that the EDSR resonance and Rabi frequencies above are expressed up to fifth order in the small energy scales $\tilde{\alpha}, \tilde{B}, \tilde{E}_{\mathrm{ac}} \ll \hbar \omega_{0}$. A detailed discussion of these results, and their comparison with numerical solutions of the timedependent Schrödinger equation, is included in Sec. IV D.

\section{MAGNETIC RESONANCE WITH A MISALIGNED ac FIELD}

In this section, using Floquet perturbation theory, we derive and discuss the properties of the fundamental (single-photon) and half-harmonic (two-photon) resonances in MR, for the spin- $1 / 2$ case. In particular, the results (2)-(7) are derived.

\section{A. Problem formulation}

We consider MR spin dynamics driven by a misaligned ac field. The Hamiltonian reads

$$
\mathcal{H}(t)=-\frac{1}{2} \tilde{\mathbf{B}}(t) \cdot \boldsymbol{\sigma}
$$

where the magnetic field has the form

$$
\tilde{\mathbf{B}}(t)=\left(\begin{array}{c}
0 \\
-\tilde{B}_{\mathrm{ac}} \cos \theta \cos \omega t \\
\tilde{B}-\tilde{B}_{\mathrm{ac}} \sin \theta \cos \omega t
\end{array}\right) .
$$

Here we introduced $\tilde{B}=g \mu_{B} B$ and $\tilde{B}_{\mathrm{ac}}=g \mu_{B} B_{\mathrm{ac}}$. Henceforth the parameters with a tilde, e.g., $\tilde{B}$, have energy dimension. The Hamiltonian in Eq. (16) has four parameters: the strength of the static field $\tilde{B}$, the strength of the driving field $\tilde{B}_{\text {ac }}$, the frequency of the driving field $\omega$, and the misalignment angle $\theta$. Note that $\theta=0$ corresponds to a transverse ac field, and $\theta=\pi / 2$ corresponds to a longitudinal ac field. We consider the case of weak driving, $\tilde{B}_{\text {ac }} \ll \tilde{B}$.

In particular, we want to solve the initial-value problem described in Sec. I: the initial state is the ground state $|\uparrow\rangle$ of the Hamiltonian without driving, i.e., $\psi(t=0)=|\uparrow\rangle$, driving is switched on abruptly at $t=0$, and we are interested in the time evolution $\psi(t)$ of this state. We calculate the transition probability describing the time-dependent occupation of the excited state $|\downarrow\rangle$ at the fundamental and half-harmonic resonances, and from those we deduce the parameter dependencies of the resonance frequencies and the Rabi frequencies.

In the rest of this section, we use Floquet perturbation theory [2,36-38] to derive the results and to provide qualitative interpretations in simple limiting cases, such as the limits of transversal and longitudinal ac fields. Even though similar treatments can be found in the literature [2,38], we present a detailed discussion of the MR problem for the following reason. The MR problem is relatively simple as compared to the EDSR problem, which can be appreciated, e.g., by comparing the driven two-level Hamiltonians of Eqs. (16) and (41), respectively. Moreover, as we will show, the Floquet method and the qualitative interpretations we describe here for the MR problem can be carried over to the EDSR problem, once the $2 \times 2$ effective qubit Hamiltonian in Eq. (41) is obtained for the latter. This allows us to provide a rather compact description of the EDSR in the forthcoming sections, by referencing this section wherever possible.

\section{B. Floquet method}

The Floquet method allows one to find the solution of an initial-value problem of a periodically driven quantum system, described by the time-periodic Hamiltonian $H(t)=$ $H(t+T)$. The period of the driving is denoted by $T$, and the corresponding (angular) frequency by $\omega=2 \pi / T$. The key ingredient of the method is the quantum-mechanical Floquet theorem [36], which guarantees that the Schrödinger equation $i \hbar \dot{\Psi}(t)=\mathcal{H}(t) \Psi(t)$ of a $d$-level system has $d$ solutions $\Psi_{k}(t)$ $(k=1, \ldots, d)$ that are themselves periodic with period $T$, apart from a phase factor. Therefore, these special solutions 
have the form

$$
\left|\Psi_{k}(t)\right\rangle=e^{-i E_{k} t / \hbar} \sum_{l=1}^{d} \sum_{m=-\infty}^{\infty} c_{k, l m} e^{i m \omega t}\left|\psi_{l}\right\rangle,
$$

where $\left|\psi_{l}\right\rangle$ is an arbitrary basis of the Hilbert space. Note that the result of the double sum is a periodic function of $t$ with period $T$. In Eq. (18), the quantity $E_{k}$ and the coefficients $c_{k, l m}$ are a priori unknown; the former is called quasienergy. Once these special solutions $\left|\Psi_{k}(t)\right\rangle$ are found, they provide the propagator

$$
U(t, 0)=\sum_{k=1}^{d}\left|\Psi_{k}(t)\right\rangle\left\langle\Psi_{k}(0)\right|,
$$

which in turn provides the solution of any initial-value problem via

$$
\Psi(t)=U(t, 0) \Psi(0) .
$$

The special solutions $\Psi_{k}(t)$ are found by using Eq. (18) as an ansatz, substituting it to the Schrödinger equation, evaluating the scalar product of the equation with $\left\langle\psi_{l^{\prime}}\right|$, multiplying the equation by $e^{-i m^{\prime} \omega t}$, and integrating the equation in time between $t=0$ and $t=T$. This procedure yields the following eigenvalue equation for $E_{k}$ :

$$
\sum_{l=1}^{d} \sum_{m=-\infty}^{\infty} \mathcal{F}_{l^{\prime} m^{\prime}, l m} c_{k, l m}=E_{k} c_{k, l^{\prime} m^{\prime}}
$$

where

$$
\mathcal{F}_{l^{\prime} m^{\prime}, l m}=m \hbar \omega \delta_{l^{\prime} l} \delta_{m^{\prime} m}+\sum_{n=-\infty}^{\infty}\left\langle\psi_{l^{\prime}}\left|\mathcal{H}^{(n)}\right| \psi_{l}\right\rangle \delta_{m^{\prime}, n+m}
$$

is the Floquet matrix or Floquet Hamiltonian, and we introduced the Fourier components $\mathcal{H}^{(n)}$ of the Hamiltonian via

$$
\mathcal{H}(t)=\sum_{n=-\infty}^{\infty} \mathcal{H}^{(n)} e^{i n \omega t}
$$

We call two eigenvalue-eigenvector pairs of $\mathcal{F}$ equivalent if the two time-dependent solutions they generate via Eq. (18) are the same. Importantly, even though the number of eigenvalueeigenvector pairs of $\mathcal{F}$ is infinite, they form only $d$ equivalence classes.

In summary, we have transformed the time-dependent Schrödinger equation of the periodically driven $d \times d$ Hamiltonian $\mathcal{H}(t)$ into the time-independent Schrödinger equation (21) of the infinite-dimensional Floquet Hamiltonian $\mathcal{F}$. To construct the special solutions (18), and thereby the solution of any initial-value problem via Eqs. (19) and (20), the quasienergies $E_{k}$ and the corresponding eigenvectors $c_{k}$ should be found by solving the eigenvalue problem of the Floquet Hamiltonian $\mathcal{F}$.

\section{Perturbative description of the transition probability}

After reviewing the Floquet method in general, we now apply this to the MR problem defined in Eq. (16). Here, we have a two-level system, therefore $d=2$, and we use $|\alpha\rangle \equiv$ $|\uparrow\rangle \equiv\left|\psi_{1}\right\rangle$ and $|\beta\rangle \equiv|\downarrow\rangle \equiv\left|\psi_{2}\right\rangle$ to denote these levels. The Fourier components of the Hamiltonian read

$$
\begin{aligned}
\mathcal{H}^{(0)} & =-\frac{1}{2} \tilde{B} \sigma_{z}, \\
\mathcal{H}^{( \pm 1)} & =\frac{1}{4} \tilde{B}_{\mathrm{ac}}\left(\cos \theta \sigma_{y}+\sin \theta \sigma_{z}\right),
\end{aligned}
$$

and the other Fourier components are zero.

First, consider the case when the drive frequency is close to the fundamental resonance, $\hbar \omega \approx \tilde{B}$. Then, the diagonal elements of the Floquet Hamiltonian $\mathcal{F}$ (Floquet levels) form pairs:

$$
\mathcal{F}_{\alpha m, \alpha m}=m \hbar \omega-\frac{1}{2} \tilde{B} \approx(m-1) \hbar \omega+\frac{1}{2} \tilde{B}=\mathcal{F}_{\beta, m-1, \beta, m-1} .
$$

Fundamental resonance

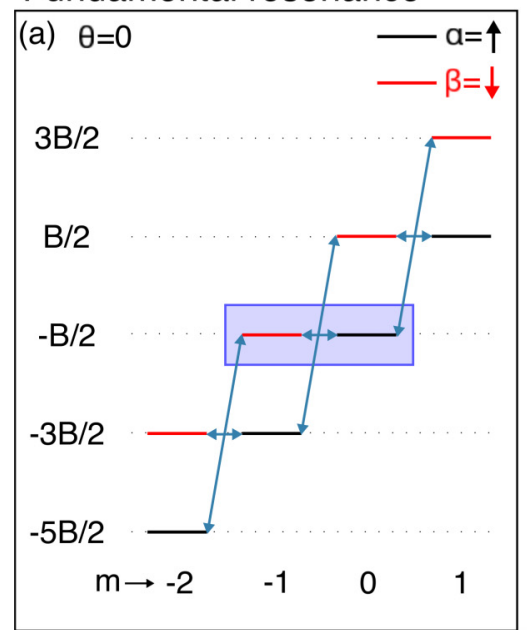

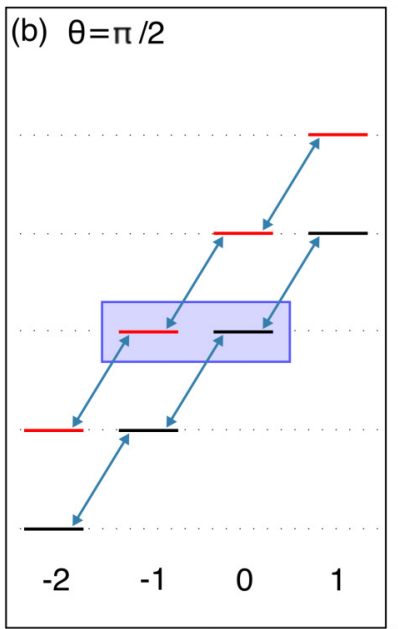

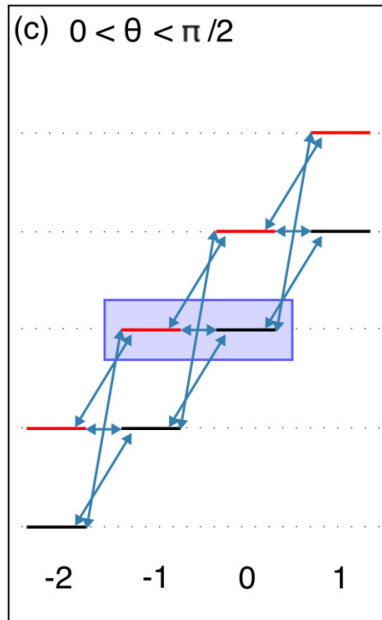

FIG. 2. (Color online) Magnetic resonance in a misaligned ac field: structure of the Floquet Hamiltonian at the fundamental resonance. Panels show cases when the ac field is perpendicular to the static field (a), is parallel to the static field (b), has finite perpendicular and parallel components (c). Horizontal lines (blue arrows) correspond to diagonal (off-diagonal) matrix elements of the Floquet Hamiltonian $\mathcal{F}$. The vertical position of each horizontal line corresponds to the value of the diagonal matrix element. 
The distance between different pairs is approximately $\hbar \omega \approx \tilde{B}$, which is much larger than the energy scale $\tilde{B}_{\text {ac }}$ characterizing the off-diagonal elements of $\mathcal{F}$. Therefore, the tools of quantum-mechanical perturbation theory can be used to provide an approximate solution of the eigenvalue problem of the Floquet Hamiltonian.

The structure of the Floquet Hamiltonian $\mathcal{F}$ is visualized for the case $\hbar \omega=\tilde{B}$ in the level diagram shown in Fig. 2 . Horizontal lines represent the diagonal matrix elements $\mathcal{F}_{l m, l m}$ of the Floquet Hamiltonian, their vertical positions correspond to their value, their color (black, red) represents their spin index $l \in(\alpha, \beta)$, and their horizontal position stands for their Floquet index $m=\ldots,-1,0,1,2, \ldots$ The vertical spacing of the Floquet levels is $\hbar \omega=\tilde{B}$. The blue arrows indicate the nonzero off-diagonal matrix elements of $\mathcal{F}$, which are of the order of $B_{\mathrm{ac}}$ and hence small compared to the level spacing.

In the case $\hbar \omega=\tilde{B}$ shown in Fig. 2, the Floquet levels form degenerate pairs. The pair formed by $\mathcal{F}_{\beta,-1, \beta,-1}$ and $\mathcal{F}_{\alpha, 0, \alpha, 0}$ is highlighted in Fig. 2 by the blue box. The subspace of this pair is weakly coupled to the other Floquet levels, hence this coupling can be treated perturbatively using (time-independent) Schrieffer-Wolff perturbation theory [39], which is also known as quasidegenerate perturbation theory [40]. This perturbative treatment is also applicable if there is a finite but small detuning $\delta=\omega-\tilde{B} / \hbar \ll \tilde{B} / \hbar$ from the resonance condition. The small dimensionless parameter characterizing the strength of the perturbation is $\epsilon=\tilde{B}_{\mathrm{ac}} / \tilde{B}$.

In this case, the Floquet Hamiltonian reads

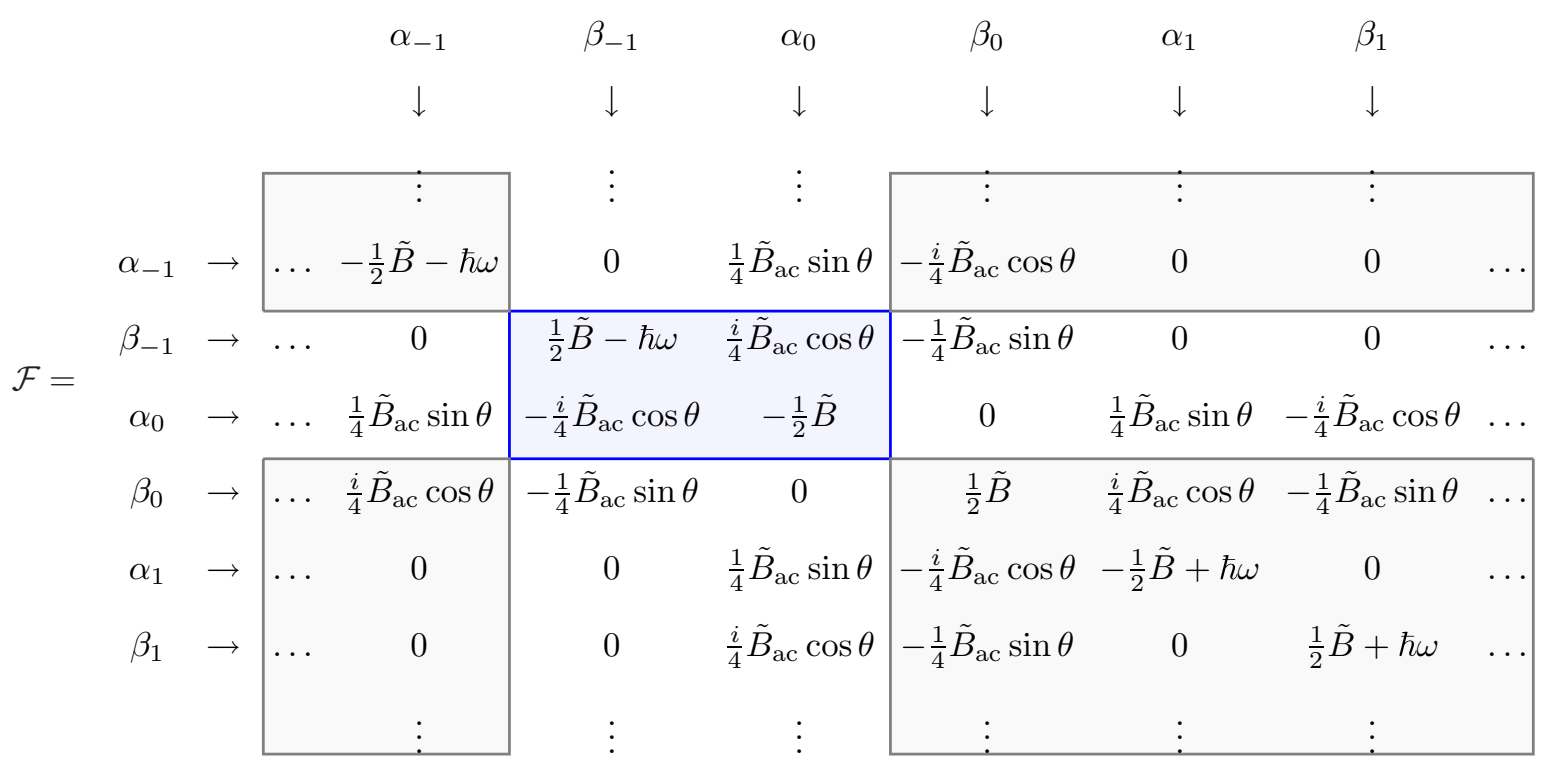

\section{Fundamental resonance within RWA}

Using first-order perturbation theory, the two nonequivalent eigenvalues and eigenvectors of $\mathcal{F}$ can be found approximately. For this we introduce $\mathcal{F}_{0}$ and $\mathcal{F}_{1}$ so that $\mathcal{F}=\mathcal{F}_{0}+\mathcal{F}_{1} . \mathcal{F}_{0}$ is the diagonal component of $\mathcal{F}$ at $\omega=\tilde{B} / \hbar$., i.e., at $\delta=0$.

First-order perturbation theory in $\mathcal{F}_{1}$ amounts to diagonalizing the $2 \times 2$ block highlighted in purple in Eq. (26) and Fig. 2, i.e. the part belonging to states $\beta_{-1}$ and $\alpha_{0}$. For future reference, we recast this $2 \times 2$ block to the form

$$
\tilde{\mathcal{F}}=\left(\begin{array}{cc}
\epsilon_{0}+\Delta & i \lambda \\
-i \lambda & \epsilon_{0}-\Delta
\end{array}\right),
$$

where $\epsilon_{0}=-\frac{1}{2}(\tilde{B}+\hbar \delta), \Delta=-\hbar \delta / 2$, and $\lambda=\frac{1}{4} \tilde{B}_{\text {ac }} \cos \theta$. The matrix $\tilde{\mathcal{F}}$ has eigenvalues

$$
\tilde{E}_{ \pm}=\epsilon_{0} \pm \sqrt{\Delta^{2}+\lambda^{2}}
$$

and corresponding eigenvectors

$$
\tilde{c}_{ \pm}=N_{ \pm}\left[\frac{i}{\lambda}\left(\Delta \pm \sqrt{\Delta^{2}+\lambda^{2}}\right), 1\right],
$$

where $N_{ \pm}$is a normalization constant. Note that instead of using the numerical index $k \in(1,2)$ labeling the solutions (18), in Eqs (28) and after we use the values $k \in(+,-)$.

The results (28) and (29) imply that the two nonequivalent approximate eigenvalue-eigenvector pairs of $\mathcal{F}$ are $\left(\tilde{E}_{ \pm}, c_{ \pm}\right)$, where

$$
c_{ \pm, l m}= \begin{cases}\tilde{c}_{ \pm, 1} & \text { if }(l, m)=(\beta,-1), \\ \tilde{c}_{ \pm, 2} & \text { if }(l, m)=(\alpha, 0), \\ 0 & \text { otherwise, }\end{cases}
$$

and $\tilde{c}_{ \pm, 1}$ and $\tilde{c}_{ \pm, 2}$ are the components of $\tilde{c}_{ \pm}$in Eq. (29). This result allows us to construct the transition probability $P_{\beta \leftarrow \alpha}(t)=|\langle\beta \mid \Psi(t)\rangle|^{2}$ from the initial spin (ground) state $|\uparrow\rangle \equiv|\alpha\rangle$ to the excited state $|\downarrow\rangle \equiv|\beta\rangle$ via Eqs. (18)-(20). A straightforward calculation yields

$$
P_{\beta \leftarrow \alpha}(t)=\frac{\lambda^{2}}{\lambda^{2}+\Delta^{2}} \sin ^{2}\left(\frac{1}{\hbar} \sqrt{\Delta^{2}+\lambda^{2}} t\right) .
$$

According to Eq. (31), the spin makes complete Rabi oscillations if $\Delta=0$, that is, $\delta=\omega-\tilde{B} / \hbar=0$. Hence the single-photon resonance frequency is $\omega_{\text {res }}^{(1)}=\tilde{B} / \hbar$. The Rabi 
frequency upon resonant driving is $\Omega_{\text {res }}^{(1)}=2 \lambda / \hbar=\frac{\tilde{B}_{\mathrm{ac}}}{2 \hbar} \cos \theta$; thus only the transverse component of the ac field contributes to the Rabi frequency at the fundamental resonance. In fact, the result (31) is equivalent to the one obtained by neglecting the longitudinal ac field and performing RWA.

\section{Fundamental resonance beyond the RWA: Bloch-Siegert shift of the resonance frequency}

Let us discuss the corrections to $\omega_{\text {res }}^{(1)}$ and $\Omega_{\text {res }}^{(1)}$ beyond the RWA. To this end, we incorporate in the analysis the effect of those matrix elements of $\mathcal{F}_{1}$ that connect the two highlighted Floquet levels [see Eq. (26) and Fig. 2] to the complementary subspace. This is done via a (time-independent) SchriefferWolff transformation that is second order in $\mathcal{F}_{1}$. The resulting effective $2 \times 2$ Floquet Hamiltonian $\tilde{\mathcal{F}}$ has the form given in Eq. (27), with

$$
\begin{aligned}
\Delta & =-\frac{1}{2} \hbar \delta+\frac{\tilde{B}_{\mathrm{ac}}^{2} \cos ^{2} \theta}{32 \tilde{B}}, \\
\lambda & =\frac{\tilde{B}_{\mathrm{ac}} \cos \theta}{4} .
\end{aligned}
$$

Recall that the eigenvalues and eigenvectors of $\tilde{\mathcal{F}}$ are given by Eqs. (28) and (29). From these, we conclude that the two nonequivalent approximate eigenvalue-eigenvector pairs of $\mathcal{F}$ are $\left(\tilde{E}_{ \pm}, c_{ \pm}\right)$, where

$$
c_{ \pm, l m}= \begin{cases}\tilde{c}_{ \pm, 1}+o\left(\epsilon^{2}\right) & \text { if }(l, m)=(\beta,-1), \\ \tilde{c}_{ \pm, 2}+o\left(\epsilon^{2}\right) & \text { if }(l, m)=(\alpha, 0), \\ o(\epsilon) & \text { otherwise. }\end{cases}
$$

We neglect the perturbative corrections $\sim o(\epsilon), o\left(\epsilon^{2}\right)$ in the eigenvectors $c_{ \pm}$, and this implies that the approximate transition probability is given by Eq. (31). Equation (31) predicts that complete Rabi oscillations are induced when $\Delta=0$; solving Eq. (32a) for $\omega$ (recall that $\delta=\omega-\tilde{B} / \hbar$ ) provides the resonance frequency shown in Eq. (2). The second term of Eq. (2) corresponds to the Bloch-Siegert shift of the resonance frequency: as the drive strength $\tilde{B}_{\mathrm{ac}}$ is increased, the resonance frequency shifts upwards. This feature is further discussed in Sec. III D. Finally, the Rabi frequency at the fundamental resonance, which is given by $\hbar \Omega_{\text {res }}^{(1)}=2 \lambda$, is expressed using Eq. (32b) in Eq. (4); the result is the same as in the RWA.

\section{Half-harmonic resonance}

Let us now consider the spin dynamics at half-harmonic resonance, when $\hbar \omega \approx \tilde{B} / 2$. The level diagram visualizing the Floquet Hamiltonian in the case $\hbar \omega=\tilde{B} / 2$ is shown in Fig. 3. Again, we can identify degenerate pairs of Floquet levels, e.g., the pair $\left(\mathcal{F}_{\beta,-1, \beta,-1}, \mathcal{F}_{\alpha, 1, \alpha, 1}\right)$ highlighted with the blue box in Fig. 3 .

Note that in this case, there is no direct matrix element (blue arrow) connecting these two Floquet levels. This implies that by repeating the first-order perturbation theory (equivalent to RWA) done in Sec. III C 1, we would conclude that two-photon Rabi oscillations do not happen. However, this result is not correct; two-photon Rabi oscillations can happen. To see that, we perform a second-order Schrieffer-Wolff transformation on $\mathcal{F}$, as done in Sec. III C 2. Furthermore we use the appropriate notation $\delta=\omega-\frac{\tilde{B}}{2 \hbar}$. The obtained effective $2 \times 2$ Floquet Hamiltonian $\tilde{\mathcal{F}}$ has the form given in Eq. (27), with

$$
\begin{aligned}
\Delta & =\hbar \delta-\frac{\tilde{B}_{\mathrm{ac}}^{2} \cos ^{2} \theta}{6 \tilde{B}}, \\
\lambda & =\frac{\tilde{B}_{\mathrm{ac}}^{2} \sin 2 \theta}{8 \tilde{B}} .
\end{aligned}
$$

Following the approach used in Sec. III C 2, and solving $\Delta=0$ we find that the half-harmonic resonance frequency $\omega_{\text {res }}^{(2)}$ is given by Eq. (5). Furthermore, using $\hbar \Omega_{\text {res }}^{(2)}=2 \lambda$ and Eq. (34b), the Rabi frequency at the half-harmonic resonance is obtained as shown in Eq. (7).

\section{Half-harmonic resonance}
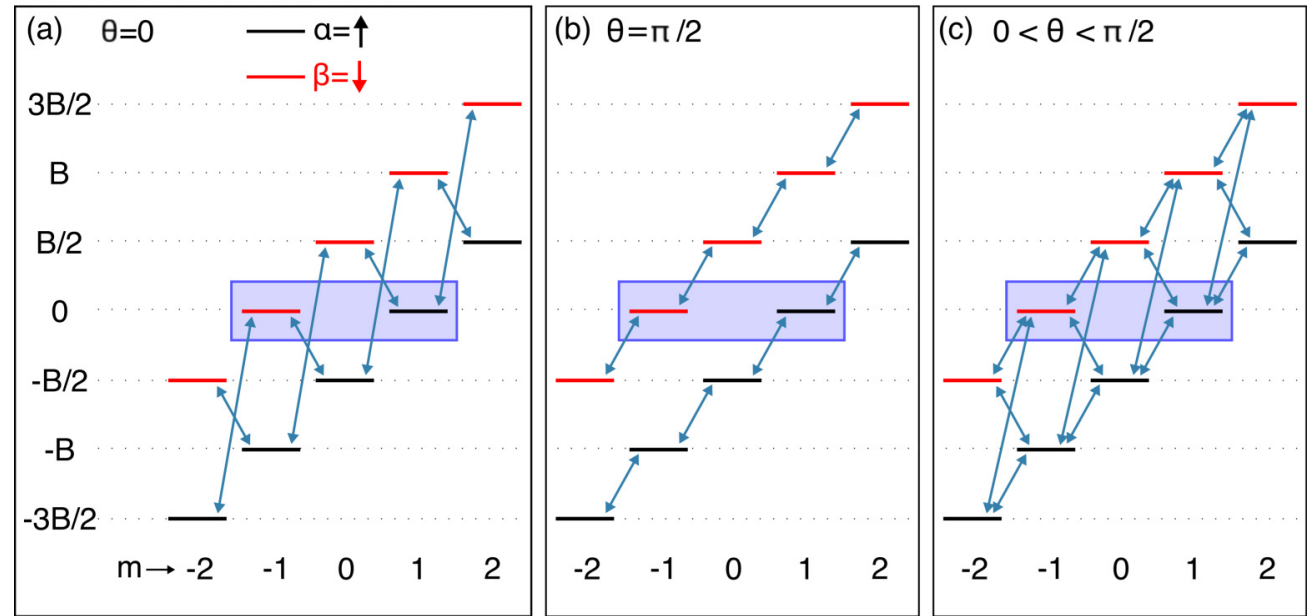

FIG. 3. (Color online) Magnetic resonance in a misaligned ac field: structure of the Floquet Hamiltonian at the half-harmonic resonance. Panels show cases when the ac field is perpendicular to the static field (a), is parallel to the static field (b), has finite perpendicular and parallel components (c). Horizontal lines (blue arrows) correspond to diagonal (off-diagonal) matrix elements of the Floquet Hamiltonian $\mathcal{F}$. The vertical position of each horizontal line corresponds to the value of the diagonal matrix element. 


\section{Discussion}

Let us now discuss the main features of the results (2), (4), (5), and (7).

Consider first the fundamental resonance frequency $\omega_{\text {res }}^{(1)}$ expressed in Eq. (2). The second term in Eq. (2) implies that $\omega_{\text {res }}^{(1)}$ has a positive drive-strength-dependent correction $\propto \tilde{B}_{\text {ac }}^{2} / \tilde{B}$ with respect to the nominal Zeeman splitting $\tilde{B}$. This correction is known as the BSS, which can be regarded as a special case of the ac Stark shift [41].

Note that the parameter $\lambda$ and hence the Rabi frequency $\Omega_{\text {res }}^{(1)}$ sets the frequency broadening of the fundamental transition, as indicated by the prefactor $\lambda^{2} /\left(\lambda^{2}+\Delta^{2}\right)$ on the right-hand side of Eq. (31). According to Eq. (4), this power broadening of the fundamental resonance is greater by a factor of $\tilde{B} / \tilde{B}_{\mathrm{ac}}$ than the BSS.

Equation (2) also shows that the BSS is finite in the limit of purely transversal drive $(\theta=0)$, and vanishes in the limit of purely longitudinal drive $(\theta=\pi / 2)$. The respective Floquet level diagrams in Figs. 2(a) and 2(b) provide a straightforward interpretation: the BSS can be regarded as a consequence of coupling-induced repulsion between the Floquet levels. In Fig. 2(a) $(\theta=0)$, the Floquet level $\mathcal{F}_{\beta,-1, \beta,-1}$ is connected by a blue arrow (off-diagonal matrix elements of $\mathcal{F}$ ) to the lower-lying Floquet level $\mathcal{F}_{\alpha,-2, \alpha,-2}$. The consequence of this coupling in second-order perturbation theory is level repulsion; i.e., the lower-lying Floquet level pushes $\mathcal{F}_{\beta,-1, \beta,-1}$ upwards. Similarly, $\mathcal{F}_{\alpha 0, \alpha 0}$ is pushed downwards by its coupling to the higher-lying Floquet level $\mathcal{F}_{\beta 1, \beta 1}$. These second-order level shifts appear in Eq. (32a) as the last term, and give rise to a finite BSS. In contrast, each of the highlighted Floquet levels in Fig. 2(b) $(\theta=\pi / 2)$ is connected to one higher-lying and one lower-lying Floquet level, and the corresponding downward and upward level repulsions cancel each other, giving rise to a vanishing BSS in this case.

Consider now the half-harmonic resonance. Equation (7) provides the corresponding Rabi frequency, and it indicates the existence of Rabi oscillations unless $\theta=0$ or $\theta=\pi / 2$. That is, Rabi oscillations appear at half-harmonic excitation only if the transversal and longitudinal components of the driving field are both nonzero. The Floquet level diagrams shown in Fig. 3 provide a visual interpretation of this feature: Rabi oscillations arise if the blue arrows (off-diagonal matrix elements of $\mathcal{F}$ ) draw at least one path between the two Floquet levels highlighted by the purple box, via virtual intermediate Floquet levels outside the box. In the special cases $\theta=0$ and $\theta=\pi / 2$ depicted in Figs. 3(a) and 3(b), respectively, no such paths exist. However, there exist infinitely many such paths for $0<\theta<\pi / 2$ [Fig. 3(c)], due to the coexistence of spin-conserving and spin-flip off-diagonal matrix elements. In particular, in our second-order Schrieffer-Wolff transformation leading to the result (7), the two two-step paths via $\mathcal{F}_{\alpha, 0, \alpha, 0}$ and $\mathcal{F}_{\beta, 0, \beta, 0}$ are incorporated.

In the case of the half-harmonic resonance, the relation between the power broadening and the BSS is qualitatively different from the case of the fundamental resonance. For the half-harmonic resonance, the power broadening is given by Eq. (7), whereas the BSS is given by the second term of Eq. (5), i.e., the two quantities are of the same order, both being $\sim \tilde{B}_{\text {ac }}^{2} / \tilde{B}$. Hence we expect that for the half-harmonic resonance, the BSS is relatively easily resolvable experimentally, at least if the dissipative frequency scales are smaller than the power broadening.

Equation (5) also shows that the BSS is finite in the limit of purely transversal excitation $(\theta=0)$, and vanishes in the limit of purely longitudinal excitation $(\theta=\pi / 2)$. An interpretation completely analogous to the case of the fundamental resonance can be given based on the Floquet level diagrams in Fig. 3(a) and $3(\mathrm{~b})$.

\section{ELECTRICALLY DRIVEN SPIN RESONANCE}

\section{A. The model}

From now on, we describe EDSR mediated by spin-orbit interaction in a $1 \mathrm{D}$ parabolic quantum dot. The setup is shown in Fig. 1. The Hamiltonian

$$
\mathcal{H}=\mathcal{H}_{0}+\mathcal{H}_{E}+\mathcal{H}_{B}+\mathcal{H}_{\text {SO }}
$$

includes the harmonic-oscillator Hamiltonian $\left(\mathcal{H}_{0}\right)$ consisting of the kinetic energy of the electron and the parabolic confinement potential, the ac electric potential arising from the driving electric field $\left(\mathcal{H}_{E}\right)$, the static Zeeman effect caused by a homogeneous magnetic field $\left(\mathcal{H}_{B}\right)$, and the spin-orbit term $\left(\mathcal{H}_{\mathrm{SO}}\right)$. The explicit forms of these terms, respectively, are as follows:

$$
\begin{aligned}
\mathcal{H}_{0} & =\frac{p_{z}^{2}}{2 m}+\frac{1}{2} m \omega_{0}^{2} z^{2}=\hbar \omega_{0}\left(a^{\dagger} a+\frac{1}{2}\right), \\
\mathcal{H}_{E} & =e z E_{\mathrm{ac}} \sin (\omega t)=\tilde{E}_{\mathrm{ac}} \sin (\omega t)\left(a^{\dagger}+a\right), \\
\mathcal{H}_{B} & =-\frac{1}{2} g^{*} \mu_{B} B \sigma_{z}=-\frac{1}{2} \tilde{B} \sigma_{z}, \\
\mathcal{H}_{\mathrm{SO}} & =\alpha p_{z} \mathbf{n}_{\mathrm{so}} \cdot \sigma=i \tilde{\alpha}\left(a^{\dagger}-a\right) \mathbf{n}_{\mathrm{so}} \cdot \sigma .
\end{aligned}
$$

Here, $a$ and $a^{\dagger}$ are the ladder operators of the harmonic oscillator Hamiltonian, and $\mathbf{n}_{\mathrm{so}}=(0, \cos \theta, \sin \theta)$ is the direction of the effective magnetic field arising from spin-orbit coupling. Furthermore, we defined

$$
\begin{gathered}
\tilde{B}=g^{*} \mu_{B} B, \\
\tilde{\alpha}=\alpha \sqrt{\frac{m \hbar \omega_{0}}{2}}, \\
\tilde{E}_{\mathrm{ac}}=e E_{\mathrm{ac}} \sqrt{\frac{\hbar}{2 m \omega_{0}}} .
\end{gathered}
$$

These quantities have the dimension of energy.

Note that we use the same notation $\theta$ for two different quantities: $\theta$ appears in Eq. (17) as the ac field misalignment angle in MR, and it also appears in this section and in Fig. 1, as the angle characterizing the direction of the spin-orbit term. We use the same notation for these quantities as they play very similar roles in the spin dynamics.

It is natural to represent the Hamiltonian terms (36) in the product basis of the orbital and spin degrees of freedom, $\left\{\left|n_{\sigma}\right\rangle \mid n=0,1,2, \ldots ; \sigma=\uparrow, \downarrow\right\}$, where $n$ is the harmonicoscillator orbital quantum number and $\sigma$ is the spin quantum number with quantization along $z$. 
We will refer to the two lowest-energy eigenstates of our static Hamiltonian $\mathcal{H}_{0}+\mathcal{H}_{B}+\mathcal{H}_{\mathrm{SO}}$ as the qubit basis states. The qubit basis state with the lower (higher) energy will be denoted by $|G\rangle(|E\rangle)$.

The electron is initialized in state $|G\rangle$ at $t=0$. Our aim is to describe the time evolution of the state upon driving. In particular, we are interested in the time-dependent occupation probability $P_{E}(t)$ of state $|E\rangle$. It is expected that at resonant driving $\hbar \omega \approx \tilde{B}$, the dynamics resembles Rabi oscillations. Subharmonic (multiphoton or $N$-photon) resonances at $\hbar \omega \approx$ $\tilde{B} / N$ (where $N=1,2, \ldots$ ) are also expected. In this work we focus on the fundamental (single-photon, $N=1$ ) and halfharmonic (two-photon, $N=2$ ) resonances.

We aim at an analytical, perturbative description of spin transitions induced by the ac electric field. In particular, we calculate the resonance frequency and the Rabi frequency at resonant driving. We consider the parameter range where the energy scale $\hbar \omega_{0}$ of the confinement potential dominates the other four energy scales, the latter ones being assumed to be comparable in magnitude:

$$
\hbar \omega \sim \tilde{\alpha} \sim \tilde{E}_{\mathrm{ac}} \sim \tilde{B} \ll \hbar \omega_{0} .
$$

This hierarchy of energy scales will allow for a perturbative description of the dynamics, with the small parameter $\epsilon \sim$ $\frac{\omega}{\omega_{0}} \sim \frac{\tilde{\alpha}}{\hbar \omega_{0}} \sim \frac{\tilde{E}_{\mathrm{ac}}}{\hbar \omega_{0}} \sim \frac{\tilde{B}}{\hbar \omega_{0}} \ll 1$.

\section{B. Effective qubit Hamiltonian}

In the EDSR problem defined in Sec. IV A, the hierarchy of the energy scales is given by Eq. (40). Because of this hierarchy, an effective time-dependent two-level Hamiltonian [see Eq. (41) below] can be derived for the qubit dynamics, using TDSW perturbation theory, which we outline in Appendix A. This qubit Hamiltonian can then be used to express the resonance frequencies $\omega_{\text {res }}^{(1)}$ and $\omega_{\text {res }}^{(2)}$, and the corresponding Rabi frequencies at these resonances, $\Omega_{\text {res }}^{(1)}$ and $\Omega_{\text {res }}^{(2)}$, corresponding to the fundamental and half-harmonic resonances, respectively see Eqs. (8), (12), (13), and (15) above.

We use the orbital-spin product basis $\left\{\left|n_{\sigma}\right\rangle \mid n=\right.$ $0,1,2, \ldots ; \sigma=\uparrow, \downarrow\}$ as the starting point of TDSW, and take the two-dimensional subspace of $\left|0_{\uparrow}\right\rangle$ and $\left|0_{\downarrow}\right\rangle$ as the relevant subspace in TDSW. We carry out a fifth-order TDSW (in the small parameter $\epsilon$ ), which is expected to describe both the fundamental and the half-harmonic resonances. The TDSW procedure yields the effective qubit Hamiltonian

$$
\tilde{\mathcal{H}}_{q} \approx \tilde{\mathcal{H}}_{q}^{(0)}+\tilde{\mathcal{H}}_{q}^{(1)}+\tilde{\mathcal{H}}_{q}^{(2)}+\tilde{\mathcal{H}}_{q}^{(3)}+\tilde{\mathcal{H}}_{q}^{(4)}+\tilde{\mathcal{H}}_{q}^{(5)},
$$

where the six terms, representing terms from different orders in the perturbation, are listed below in Eq. (42). Note that the terms $\tilde{\mathcal{H}}_{q}^{(0)}, \tilde{\mathcal{H}}_{q}^{(2)}$, and $\tilde{\mathcal{H}}_{q}^{(4)}$ are proportional to the $2 \times 2$ unit matrix $\sigma_{0}$, therefore they do not influence the dynamics, and hence we disregard them in the forthcoming calculations; nevertheless we include them here for completeness:

$$
\begin{aligned}
& \tilde{\mathcal{H}}_{q}^{(0)}=\frac{\hbar \omega_{0}}{2} \sigma_{0}, \\
& \tilde{\mathcal{H}}_{q}^{(1)}=-\frac{\tilde{B}}{2} \sigma_{3},
\end{aligned}
$$

$$
\begin{aligned}
\tilde{\mathcal{H}}_{q}^{(2)}= & -\frac{\tilde{\alpha}^{2}+\tilde{E}_{\mathrm{ac}}^{2} \sin ^{2}(\omega t)}{\hbar \omega_{0}} \sigma_{0} \\
\tilde{\mathcal{H}}_{q}^{(3)}= & -\frac{\tilde{B} \tilde{E}_{\mathrm{ac}} \tilde{\alpha} \cos \theta}{\hbar^{2} \omega_{0}^{2}} \sin (\omega t) \sigma_{1} \\
& -\frac{\tilde{\alpha} \cos \theta}{\hbar^{2} \omega_{0}^{2}}\left[\tilde{E}_{\mathrm{ac}} \hbar \omega \cos (\omega t)+\tilde{B} \tilde{\alpha} \sin \theta\right] \sigma_{2} \\
& +\frac{\tilde{\alpha}}{\hbar^{2} \omega_{0}^{2}}\left[\tilde{B} \tilde{\alpha} \cos ^{2} \theta-\tilde{E}_{\mathrm{ac}} \hbar \omega \sin \theta \cos (\omega t)\right] \sigma_{3} \\
\tilde{\mathcal{H}}_{q}^{(4)}= & -\frac{(\tilde{B} \tilde{\alpha} \cos \theta)^{2}}{\hbar^{3} \omega_{0}^{3}} \sigma_{0} \\
\tilde{\mathcal{H}}_{q}^{(5)}= & -\left(h_{x}^{(5)} \sigma_{x}+h_{y}^{(5)} \sigma_{y}+h_{z}^{(5)} \sigma_{z}\right)
\end{aligned}
$$

In Eq. (42f), we used

$$
\begin{aligned}
h_{x}^{(5)}= & \frac{\tilde{B} \tilde{\alpha} \tilde{E}_{\mathrm{ac}} \cos \theta}{\hbar^{4} \omega_{0}^{4}}\left(2 \tilde{\alpha}^{2}-\tilde{B}^{2}\right) \sin (\omega t), \\
h_{y}^{(5)}= & \frac{\tilde{E}_{\mathrm{ac}} \tilde{\alpha} \omega^{3} \cos \theta}{\hbar \omega_{0}^{4}} \cos (\omega t) \\
& +\frac{\tilde{B} \tilde{\alpha}^{2} \sin 2 \theta}{2 \hbar^{4} \omega_{0}^{4}}\left[\tilde{B}^{2}-\tilde{\alpha}^{2}+\tilde{E}_{\mathrm{ac}}^{2} \sin ^{2}(\omega t)\right], \\
h_{z}^{(5)}= & \frac{\tilde{E}_{\mathrm{ac}} \tilde{\alpha} \omega^{3} \sin \theta}{\hbar \omega_{0}^{4}} \cos (\omega t) \\
& +\frac{\tilde{B} \tilde{\alpha}^{2} \cos ^{2} \theta}{\hbar^{4} \omega_{0}^{4}}\left[\tilde{B}^{2}-\tilde{\alpha}^{2}+\tilde{E}_{\mathrm{ac}}^{2} \sin ^{2}(\omega t)\right] .
\end{aligned}
$$

Note that the upper index in, e.g., $\tilde{\mathcal{H}}_{q}^{(3)}$ refers to the order of perturbation theory in which the term appears.

Out of the six terms in Eq. (41), $\tilde{\mathcal{H}}_{q}^{(0)}$ and $\tilde{\mathcal{H}}_{q}^{(1)}$ are simply the projected parts of $\mathcal{H}_{0}$ and $\mathcal{H}_{1} \equiv \mathcal{H}_{E}+\mathcal{H}_{B}+\mathcal{H}_{\text {SO }}$, respectively. $\tilde{\mathcal{H}}_{q}^{(2)}$ contains a static and a time-dependent second-order energy shift, due to the spin-orbit interaction and the ac electric field, respectively. $\tilde{\mathcal{H}}_{q}^{(3)}$ has five terms. The first, second, and fifth terms are spin- and time-dependent, hence these all contribute to the qubit dynamics. The third and fourth terms are static; they describe the spin-orbit-induced $g$-tensor renormalization. The fourth-order term $\tilde{\mathcal{H}}_{q}^{(4)}$ of the qubit Hamiltonian, being diagonal, does not influence spin dynamics. The static parts of the fifth-order term $\tilde{\mathcal{H}}_{q}^{(5)}$ describe higher-order $g$-tensor renormalization (those proportional to $\tilde{\alpha}^{4} \tilde{B}$ ), or nonlinear Zeeman splitting (those proportional to $\left.\tilde{\alpha}^{2} \tilde{B}^{3}\right)$.

Already at this point, there are reasons to expect that in this EDSR model, a half-harmonic resonance occurs, and that the half-harmonic resonance frequency is driving-strength dependent: (i) The third-order effective Hamiltonian $\tilde{\mathcal{H}}_{q}^{(3)}$ incorporates both longitudinal and transverse ac components, in analogy with the case of the misaligned-field MR discussed in Sec. III. (ii) The fifth-order effective Hamiltonian $\tilde{\mathcal{H}}_{q}^{(5)}$ incorporates terms proportional to $\tilde{E}_{\mathrm{ac}}^{2} \sin ^{2} \omega t=\frac{1}{2} \tilde{E}_{\mathrm{ac}}^{2}(1-\cos 2 \omega t)$. The longitudinal static part $\propto \tilde{E}_{\mathrm{ac}}^{2} \sigma_{z}$ can be interpreted as a drive-strength-dependent effective $g$-tensor renormalization, which contributes to the BSS, whereas the dynamical part 
$\propto \tilde{E}_{\mathrm{ac}}^{2} \cos 2 \omega t \sigma_{y}$ is expected to drive Rabi oscillations at half-harmonic excitation, i.e., when $2 \hbar \omega \approx \tilde{B}$.

We note that the effective Hamiltonian in Eq. (42) fulfills the expectation that no spin transition occurs if the external $B$ field and the spin-orbit field are aligned, i.e., when $\theta=\pi / 2$.

\section{Floquet perturbation theory for EDSR}

We apply Floquet perturbation theory, outlined in Sec. III C, to describe the fundamental and half-harmonic resonances. In particular, we derive the parameter dependence of the corresponding resonance frequencies $\omega_{\text {res }}^{(1)}$ and $\omega_{\text {res }}^{(2)}$, as well as the Rabi frequencies $\Omega_{\text {res }}^{(1)}$ and $\Omega_{\text {res }}^{(2)}$, at these two resonances, up to terms of the order of $\sim \tilde{B} \epsilon^{4}$. There are two significant differences in the derivation of the EDSR results with respect to that of the MR results; we outline these differences in the following.

(1) The MR Hamiltonian (16) has a driving term that is proportional to $\sin \omega t$. In contrast, the effective qubit Hamiltonian (41) we obtained for EDSR has $\cos \omega t$ terms as well as second-harmonic terms proportional to $\cos 2 \omega t$. In practice, the latter fact implies that the Floquet matrix will contain off-diagonal matrix elements that connect Floquet levels with next-nearest-neighbor Floquet quantum numbers.

(2) In the EDSR case, we repeat the same second-order time-independent Schrieffer-Wolff transformation on the Floquet Hamiltonian $\mathcal{F}$ that we applied in Secs. III $\mathrm{C} 2$ and III C 3. The Floquet Hamiltonian itself contains terms of the order of $\tilde{B}, \tilde{B} \epsilon^{2}$, and $\tilde{B} \epsilon^{4}$, since it is constructed from the effective qubit Hamiltonian that is itself the result of a finite-order perturbative calculation. When we separate the Floquet Hamiltonian to diagonal $\left(\mathcal{F}_{0}\right)$ and off-diagonal $\left(\mathcal{F}_{1}\right)$ components, and apply time-independent Schrieffer-Wolff transformation up to second order in $\mathcal{F}_{1}$, the resulting $2 \times 2$ effective Floquet Hamiltonian will involve higher-order terms, up to $\tilde{B} \epsilon^{8}$. As our original Hamiltonian was accurate only up to the $\sim \tilde{B} \epsilon^{4}$ terms, we drop the terms that are of higher order than $\sim \tilde{B} \epsilon^{4}$ from the effective Floquet Hamiltonian.

\section{Analytical versus numerical solution}

The results we obtain from Floquet perturbation theory are shown in Sec. II as Eqs. (8)-(15). In the rest of this subsection we discuss these results and compare them to numerical results.

The terms describing the fundamental resonance frequency in Eq. (8) are interpreted as nominal Zeeman splitting, $g$ tensor renormalization, nonlinear Zeeman effect, and BSS, respectively. We call the last term a BSS as it is a powerdependent correction to the resonance frequency, that is second order in the drive amplitude, hence analogous to the BSS in MR. Remarkably, the BSS in Eq. (8) is a negative correction, whereas the BSS in MR is always positive. The last term of the half-harmonic resonance frequency [Eq. (13)] is also interpreted as a BSS. Further similarities with the MR case: (i) For the fundamental resonance, the BSS is smaller $\left(\sim \tilde{B} \epsilon^{4}\right)$ than the power broadening, the latter being given by $\hbar \Omega_{\text {res }}^{(1)} \sim$ $\tilde{B} \epsilon^{2}$. (ii) For the half-harmonic resonance, the BSS, being $\sim \tilde{B} \epsilon^{4}$, is comparable to the power broadening, the latter being given by $\hbar \Omega_{\text {res }}^{(2)} \sim \tilde{B} \epsilon^{4}$. (iii) The BSS for both the fundamental and the half-harmonic resonance is proportional to $\cos ^{2} \theta$, i.e., it vanishes in the limit of purely longitudinal excitation, and finite for purely transversal excitation. These features can be explained by the argument provided in Sec. III D for the case of MR, applied to the effective qubit Hamiltonian (41).

Regarding the results (8) and (13) for the resonance frequencies, we note that their ratio is exactly 2 in the limit of vanishing driving power, i.e., $\lim _{\tilde{E}_{\mathrm{ac}} \rightarrow 0}\left(\frac{\omega_{\mathrm{res}}^{(1)}}{\omega_{\mathrm{res}}^{(2)}}\right)=2$.

We have checked that the result (8) for the fundamental Rabi frequency $\Omega_{\text {res }}^{(1)}$ matches the corresponding result of Ref. [5]; see Appendix B for details.

The analytical results are tested against numerically exact solutions of the time-dependent Schrödinger equation defined by the Hamiltonian $\mathcal{H}$ in Eq. (35). The numerical results were obtained using the truncated Hilbert space spanned by the 8 lowest-energy eigenstates of $\mathcal{H}_{0}+\mathcal{H}_{B}$, corresponding to the 4 lowest-lying levels of the harmonic oscillator. We have checked that there was no visible change in the numerical results upon extending the Hilbert space with further, higherlying orbitals.

In Fig. 4, we plot the numerically computed time evolution of the occupation probability of the excited state $|E\rangle$, for a finite range of the driving frequency in the vicinity of the "nominal" half-harmonic resonance frequency $\hbar \omega / \tilde{B}=0.5$ (see caption for parameter values). The analytical result (13) predicts complete Rabi oscillations at $\omega=\omega_{\text {res }}^{(2)}=0.4809 \tilde{B} / \hbar$, and the Rabi frequency at this resonance is predicted by Eq. (15) to be $\Omega_{\text {res }}^{(2)} \approx \frac{1}{625} \frac{\tilde{B}}{\hbar}$. These predictions are in line with the numerical data shown in Fig. 4. For a finite detuning from the resonance frequency, the Rabi oscillations become faster and reduced (i.e., they do not reach $P_{E}=1$ ), leading to the characteristic chevron pattern [4,21] known from MR. The

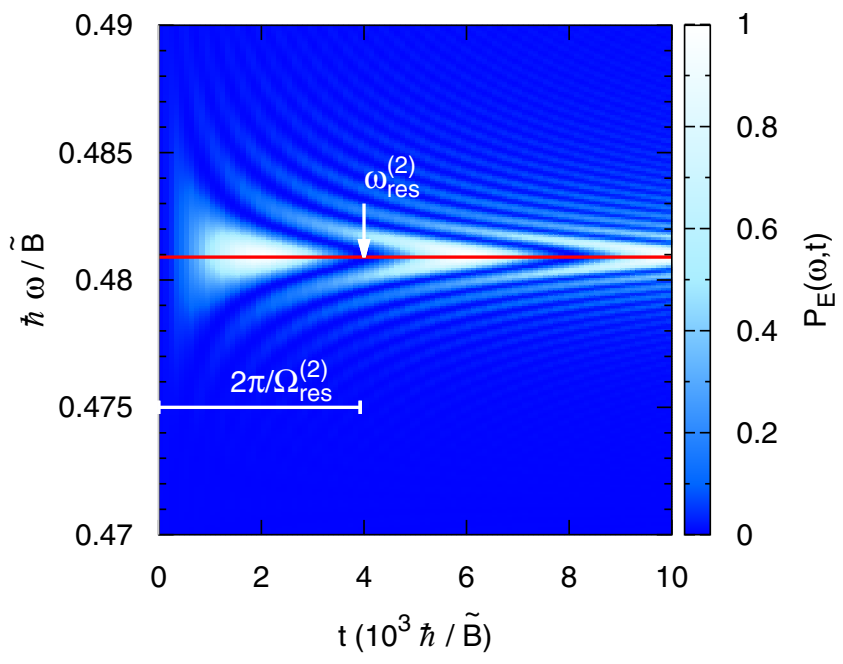

FIG. 4. (Color online) Electrically driven Rabi spin dynamics at half-harmonic resonance. The excited-state occupation probability $P_{E}$ is shown as a function of the drive frequency $\omega$ and time $t$; the numerical data reveal the chevron pattern characteristic of magnetic resonance. Parameters: $\theta=\pi / 4, \tilde{\alpha} / \tilde{B}=\tilde{E}_{\mathrm{ac}} / \tilde{B}=1, \hbar \omega_{0} / \tilde{B}=5$. The analytical results for the half-harmonic resonance frequency $\omega_{\text {res }}^{(2)}$ and the Rabi frequency $\Omega_{\text {res }}^{(2)}$ are also displayed. For the above parameter values, the latter one is related to the time period of the oscillation via $2 \pi / \Omega_{\text {res }}^{(2)}=2 \pi \times 625 \hbar / \tilde{B}$. 


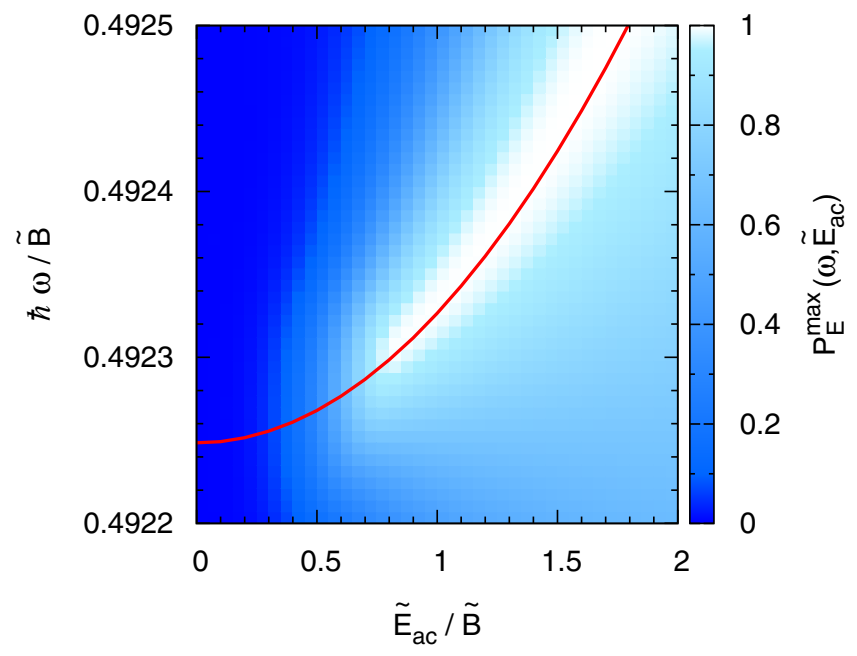

FIG. 5. (Color online) Bloch-Siegert shift and power broadening of the half-harmonic resonance. The maximal excited-state occupation probability $P_{E}^{\max }$ is shown as a function of the amplitude $\tilde{E}_{\text {ac }}$ of the driving ac electric field and drive frequency $\omega$. Parameters: $\theta=\pi / 4$, $\tilde{\alpha} / \tilde{B}=1$, and $\hbar \omega_{0} / \tilde{B}=8$. The red line indicates the analytical result for the resonance frequency as the function of electric field based on Eq. (13).

results of Fig. 4 therefore reveal simple Rabi dynamics at the half-harmonic resonance.

The density plot of Fig. 5 is a visual demonstration of the BSS, i.e., of that the resonance frequency increases with increasing drive strength. The figure shows the maximum $P_{E}^{\max }$ of the excited-state probability $P_{E}(t)$ within a time span exceeding the Rabi period at the half-harmonic resonance, as a function of the amplitude $E_{\text {ac }}$ of the ac electric field and the drive frequency $\omega$. (See caption for parameters.) Therefore,

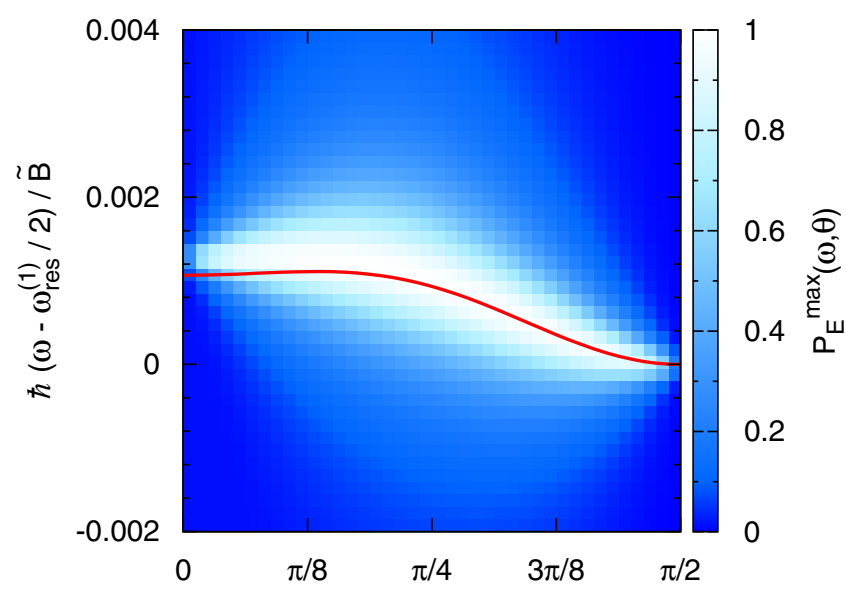

$\theta$

FIG. 6. (Color online) Anisotropy of the half-harmonic resonance. The maximal excited-state occupation probability $P_{E}^{\max }$ is shown as a function of the angle $\theta$ characterizing the spin-orbit interaction and the drive frequency $\omega$ measured from $\omega_{\text {res }}^{(1)} / 2$; see Eq. (8). The solid line corresponds to the analytically obtained half-harmonic resonance frequency; i.e., it shows $\omega_{\text {res }}^{(2)}-\frac{\omega_{\text {res }}^{(1)}}{2}$. [See Eq. (13)]. Parameters: $\tilde{E} / \tilde{B}=1, \tilde{\alpha} / \tilde{B}=1$, and $\hbar \omega_{0} / \tilde{B}=5$. vertical cuts of the density plot correspond to resonance curves. The solid line represents the analytical result (13) for the half-harmonic resonance frequency. The agreement between the analytical curve and the $P_{E}^{\max } \approx 1$ ridge of the numerical simulation reassures the validity and correspondence of the two approaches. Importantly, in Fig. 5, the BSS is comparable in magnitude to the power broadening, which makes the BSS relatively easily resolvable in experiments realizing the model we use.

A further question is how the BSS depends on the angle $\theta$ characterizing the direction of the spin-orbit interaction. This dependence is exemplified by Fig. 6, which shows $P_{E}^{\max }$ as a function of $\theta$ and the drive frequency. The latter is measured from half of the calculated fundamental resonance frequency $\omega_{\text {res }}^{(1)}$; see Eq. (8). The solid line, showing good agreement with the center of the bright $P_{E}^{\max } \approx 1$ region of the underlying density plot, shows the analytical result for the half-harmonic resonance frequency $\omega_{\text {res }}^{(2)}$ [Eq. (13)].

\section{DISCUSSION AND CONCLUSIONS}

(1) We provide a numerical example to estimate orders of magnitudes of the EDSR resonance shifts and Rabi frequencies. Let us take $B=1.7 \mathrm{~T}$ with the electronic $g$ factor 2 , yielding $\tilde{B} \approx 0.1 \mathrm{meV}$. We set $\tilde{\alpha}=0.1 \mathrm{meV}$ and $\tilde{E}_{\mathrm{ac}}=0.1 \mathrm{meV}$, and the orbital level spacing is chosen to be $\hbar \omega_{0}=1 \mathrm{meV}$. Then the order of magnitude of the Rabi frequency at the fundamental resonance becomes $\Omega_{\text {res }}^{(1)} \sim$ $\tilde{B} \epsilon^{2} / \hbar \approx 1.5 \times 10^{9} \frac{1}{\mathrm{~s}}$ corresponding to a spin-flip time of $\approx 4.3 \mathrm{~ns}$. For the half-harmonic resonance, $\Omega_{\text {res }}^{(2)} \sim \tilde{B} \epsilon^{4} / \hbar \approx$ $1.5 \times 10^{7} \frac{1}{\mathrm{~s}}$, corresponding to a spin-flip time of $\approx 430 \mathrm{~ns}$. For both resonances, the BSS is comparable to the value of $\Omega_{\text {res }}^{(2)}$ estimated above.

(2) The results presented in this work describe a perturbative regime where spin-orbit interaction is assumed to be "weak" in the sense that the spin-orbit energy scale in the QD is dominated by the QD level spacing, $\tilde{\alpha} \ll \hbar \omega_{0}$. In nanowire QD host materials such as InAs [25] and InSb [17], spin-orbit interaction is known to be "strong" in the sense that it creates a strong $g$-factor renormalization, already in the bulk materials. A question arising from these facts is, are typical InAs and InSb nanowire QDs within the range of validity of our perturbative theory? One way to answer this question is via a comparison of the dependence of the fundamental EDSR resonance frequency obtained from the perturbative theory and from experiments. The experiments [17,25] have found that the fundamental resonance frequency shows a similar angular dependence as the perturbative result Eq. (8); i.e., for a magnetic field with a fixed magnitude, the resonance frequency is maximal if the magnetic field is aligned along a certain direction and minimal if it is aligned perpendicular to that direction. To be specific, we take the data given in the first row of Table I of Ref. [25], which indicates that the ratio of the minimal and maximal resonance frequencies in the considered case was $\approx 0.84$. Using the first two terms in Eq. (8), we can identify that ratio with $1-2 \tilde{\alpha}^{2} / \hbar^{2} \omega_{0}^{2}$, yielding $\tilde{\alpha} / \hbar \omega_{0} \approx 0.28$ for this particular InAs device. A similar analysis of the experimental data in Fig. 3(c) of Ref. [17] results in an estimate $\tilde{\alpha} / \hbar \omega_{0} \approx 0.37$ for the measured InSb device. These estimates suggest that the 
InAs and InSb QDs are on the border between "weak" and "strong" spin-orbit interaction.

(3) To our knowledge, three experiments have reported subharmonic EDSR resonances in semiconductor nanowire QDs, where our model based on the Rashba-type spin-orbit interaction could be appropriate to describe the spin dynamics. The strong subharmonic resonances reported in Stehlik et al. [26] are described by a theory developed for strongly driven double quantum dots [34]. Faint half-harmonic resonances are visible in the data of Refs. [25] [see Fig. 2(b) therein] and [17] [see Fig. 2(b) therein]. A quantitative experimental analysis exploring the parameter dependencies of the corresponding resonance and Rabi frequencies would allow for a comparison with our predictions.

(4) One of our conclusions was that the BSS of the fundamental EDSR resonance frequency has an anomalous, negative sign; see Eq. (8). Here, we provide a simple physical picture explaining this result, using the unitary transformation applied in Ref. [42]. For simplicity, we focus on the case when the spin-orbit field is perpendicular to the magnetic field; i.e., $\theta=0$. Then, the unitary transformation $S$ of Ref. [42] (not to be confused with the generator of the SchriefferWolff transformation in Appendix A) applied on our static Hamiltonian $\mathcal{H}_{0}+\mathcal{H}_{B}+\mathcal{H}_{\text {SO }}$ eliminates the spin-orbit term and transforms the homogeneous magnetic field $H_{B}$ to an inhomogeneous, spiral-like magnetic field, $H_{B}^{\prime} \equiv S H_{B} S^{\dagger} \propto$ $\tilde{B}\left[\sigma_{z} \cos (z / \xi)-\sigma_{x} \sin (z / \xi)\right]$, where $\xi \propto 1 / \tilde{\alpha}$ is the spin-orbit length (see Eq. (2) of Ref. [42]). The driving electric field, incorporated in our model as $\mathcal{H}_{E}$, induces a spatial oscillation $z(t)=-A \sin \omega t$ of the electron's center of mass with an amplitude $A \propto \tilde{E}_{\text {ac }}$. Inserting this time-dependent $z(t)$ to the above expression for $H_{B}^{\prime}$, and expanding the terms up to second order in $A / \xi$, we find $H_{B}^{\prime}(t) \propto \tilde{B}\left[\sigma_{z}\left(1-\frac{A^{2}}{\xi^{2}} \sin ^{2} \omega t\right)+\right.$ $\left.\sigma_{x} \frac{A}{\xi} \sin \omega t\right]$. That is, the time-averaged $z$ component of the time-dependent magnetic field in $H_{B}^{\prime}(t)$ acquires a correction proportional to $-\tilde{B} \frac{A^{2}}{\xi^{2}} \propto-\tilde{B} \tilde{E}_{\mathrm{ac}}^{2} \tilde{\alpha}^{2}$. Notice that this correction is negative and has the same parameter dependence as the BSS in the last term of Eq. (8).

(5) To our knowledge, BSS has not yet been experimentally or theoretically analyzed in the context of EDSR. However, we wish to point out that certain numerical results in Ref. [10], related to EDSR in a double quantum dot, are reminiscent of the BSS. Figures 4(a) and 4(b) in Ref. [10] show spin Rabi oscillations for different drive strengths. Therein, the drive strength is characterized by the dimensionless quantity $f$. In Figs. 4(a) and 4(b) of Ref. [10] it is shown that the complete Rabi oscillations at the fundamental resonance become incomplete upon increasing the drive strength from $f=0.02$ to $f=0.15$, while the driving frequency is maintained. This phenomenology is reminiscent of the effect of BSS: when the drive strength is increased, BSS provides a shift of the resonance frequency, hence a fixed drive frequency becomes off-resonant, and the Rabi-oscillation amplitude decreases. It is therefore tempting to interpret these results as consequences of BSS. However, the phenomenology of BSS would imply that (i) upon further increase of the drive strength, e.g., at $f=0.35$, the amplitude of the Rabi oscillation further decreases, and (ii) the Rabi oscillation speeds up gradually as $f$ is increased from 0.02 to 0.15 and to 0.35 . The results shown in Figs. 4(a) and 4(b) of Ref. [10] disagree with these expectations, hence we conclude that the BSS phenomenology is insufficient to describe the numerical results of Ref. [10]. Importantly, Ref. [10] considers parameter settings where the undriven system consists of four approximately equidistant levels [see their Fig. 2(a)], which is a key difference with respect to the effectively two-level setup considered in our present work, and can be responsible for the phenomenology deviating from that of the BSS.

(6) Even though EDSR experiments can be performed on single QDs [13,21], many current experiments use the Pauli blockade setup for initialization and readout [3]. The latter setup consists of a double QD which is occupied by two electrons (in the simplest case) during EDSR. The inherent anharmonicity of the double-QD confinement potential, as well as the presence of the Coulomb interaction between the two (or more) electrons, can provide alternative nonlinear EDSR mechanisms [7,10,24,26,29,34], which compete with those presented in our work focusing on harmonic confinement and single-electron dynamics. For example, an apparently well understood [34] case when the two-electron and double-QD features dominate the subharmonic EDSR resonances is the experiment of Ref. [26].

In conclusion, we have studied the characteristics of EDSR in a 1D QD model with parabolic confinement, homogeneous Rashba spin-orbit interaction, and homogeneous driving electric field. We demonstrated the existence of subharmonic (multiphoton) resonances in this model, and analyzed the half-harmonic (two-photon) resonance in detail. We have analytically described the parameter dependence of the fundamental resonance frequency and the half-harmonic resonance frequency, and demonstrated that these resonance frequencies increase with increasing drive strength. This effect is analogous to the BSS in MR.

Our results describe a perturbative regime, where the orbital level spacing of the QD dominates the energy scales of the external magnetic field, spin-orbit interaction, and electrical drive. Therefore our results have direct experimental relevance for QDs with weak spin-orbit interaction. They can also serve as benchmarks for numerical studies departing from the perturbative regime. The model used here contains only minimal ingredients necessary to describe EDSR, suggesting that the subharmonic resonances and the BSS discussed here are generic features of electrically driven spin dynamics.

Note added. Recently we became aware of a related experiment [43] revealing half-harmonic EDSR in a single QD, mediated by an inhomogeneous magnetic field.

\section{ACKNOWLEDGMENTS}

We thank P. Scarlino and G. Széchenyi for useful discussions. We acknowledge funding from the EU Marie Curie Career Integration Grant CIG-293834 (CarbonQubits), the Hungarian OTKA Grants PD 100373 and 106047, and the EU ERC Starting Grant CooPairEnt 258789. G.B. acknowledges funding from the Deutsche Forschungsgemeinschaft (DFG) within SFB767 and from the EU Marie Curie Project No. ITN S3NANO. A.P. is supported by the János Bolyai Scholarship of the Hungarian Academy of Sciences. 

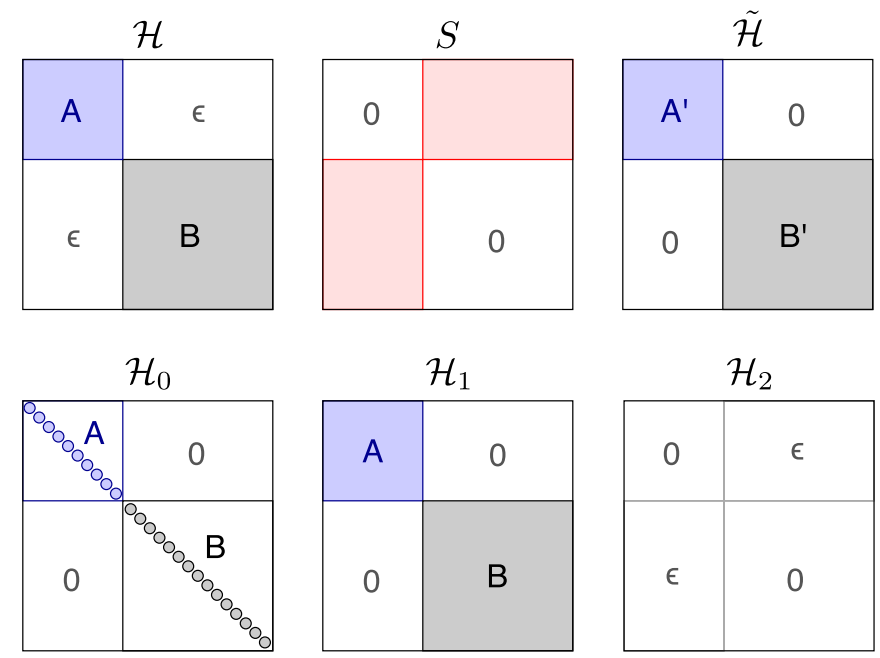

FIG. 7. (Color online) Schematic form of the matrices we encounter during the SW perturbation theory. The label $\epsilon$ refers to blocks with matrix elements that are much smaller than the energy difference between the relevant and irrelevant subspaces. In the EDSR problem, the energy scale of the those blocks is $\tilde{B} \sim \epsilon \hbar \omega_{0}$.

\section{APPENDIX A: TIME-DEPENDENT SCHRIEFFER-WOLFF PERTURBATION THEORY}

Here we introduce the time-dependent Schrieffer-Wolff perturbation theory (TDSW), the method we use to derive the effective $2 \times 2$ time-dependent Hamiltonian (41) governing the dynamics of the qubit.

Let us first recall the basic idea of standard timeindependent Schrieffer-Wolff (SW) perturbation theory $[39,40]$. We consider a Hamiltonian $\mathcal{H}=\mathcal{H}_{0}+\mathcal{H}^{\prime}$ where $\mathcal{H}_{0}$ is diagonal and $\mathcal{H}^{\prime}$ is the perturbation. Furthermore, the basis states of $\mathcal{H}$ can be divided into relevant $\mathrm{A}$ and irrelevant $B$ subspaces that have well separated energy scales. $A$ and $B$ are weakly interacting; i.e., the matrix elements connecting them are small compared to the energy separation of the two subspaces. Ideally, we can introduce a unitary transformation $e^{-S}$ that brings $\mathcal{H}$ into a block diagonal form $\tilde{\mathcal{H}}=e^{-S} \mathcal{H} e^{S}$ where the relevant and irrelevant subspaces are separated as illustrated in Fig. 7. However, in most of the cases we do not know the explicit form of the transformation $e^{-S}$ so we have to construct it bit-by-bit until the elements connecting the two subsets vanish up to the desired order of perturbation. This is usually done by expanding $e^{-S}$ in a series and constructing the terms of different orders successively.
A great advantage of SW with respect to conventional perturbation theory is that here we do not need to distinguish between the degenerate and nondegenerate cases.

Now we introduce the time-dependent SW perturbation theory as a natural extension of the time-independent case. Similar approaches have been applied for particular problems in Refs. [44-46]; here, we provide a general description of the method, which we utilized in the main text for deriving the effective qubit Hamiltonian (41) of the EDSR problem.

Consider the time-dependent Hamiltonian $\mathcal{H}(t)=\mathcal{H}_{0}+$ $\mathcal{H}^{\prime}(t)$, where the perturbation is divided into a block-diagonal and block-off-diagonal part $\mathcal{H}^{\prime}(t)=\mathcal{H}_{1}+\mathcal{H}_{2}$ as shown in Fig. 7.

In our problem (see Sec. IV A), $\mathcal{H}_{1}=\mathcal{H}_{B}$ and $\mathcal{H}_{2}(t)=$ $\mathcal{H}_{E}(t)+\mathcal{H}_{\text {So }}$. Note that there $\mathcal{H}_{1}$ happens to be a timeindependent perturbation, but the treatment outlined here is readily applicable to a time-dependent block-diagonal perturbation as well.

Similarly to the SW we successively build the unitary transformation $U(t)=e^{-S(t)}$ that separates the subspaces A and $\mathrm{B}$, but here the matrix $S(t)$ is now time-dependent. Note that any unitary transformation can be written in this form, and the matrix $S(t)$ should be anti-Hermitian to ensure the unitary character of $U(t)$. The matrix $S(t)$ is chosen to be block-off-diagonal (see Fig. 7). Note also that because of the weakness of the inter-subspace coupling, the unitary transformation $U(t)$ is close to unity, and hence $S(t)$ is small and can be expressed as a power series with respect the perturbing terms.

The transformation of time-dependent Schrödinger equation $-i \hbar \frac{\partial}{\partial t} \psi(t)+\mathcal{H}(t) \psi(t)=0$ with the above $U(t)$ is canonical; i.e., it preserves the form of the time evolution equation. The transformed wave function and Hamiltonian read as

$$
\begin{gathered}
\tilde{\psi}(t)=e^{-S(t)} \psi(t), \\
\tilde{\mathcal{H}}(t)=e^{-S(t)} \mathcal{H}(t) e^{S(t)}+i \hbar \frac{\partial e^{-S(t)}}{\partial t} e^{S(t)} .
\end{gathered}
$$

From now on, we might suppress the time argument and denote time derivatives such as $\frac{\partial}{\partial t} \psi$ as $\dot{\psi}$.

Starting from Eq. (A2), we utilize the power series of the exponential function. The second term in Eq. (A2) is the heart of the time-dependent SW transformation; in the timeindependent case this term vanishes as $S$ is time-independent. The expansion of the first term in Eq. (A2) is known from SW formalism, therefore we do not discuss it here. The explicit form of the second term, after expanding the exponential function, has the following form:

$$
\begin{aligned}
\frac{\partial e^{-S}}{\partial t} e^{S} & =\left[\frac{\partial}{\partial t}\left(-S+\frac{1}{2 !} S^{2}-\frac{1}{3 !} S^{3}+\cdots\right)\right]\left(I+S+\frac{1}{2 !} S^{2}+\frac{1}{3 !} S^{3}+\cdots\right) \\
& =\left(-\dot{S}+\frac{1}{2 !} \dot{S} S+\frac{1}{2 !} S \dot{S}-\frac{1}{3 !} \dot{S} S^{2}-\frac{1}{3 !} S \dot{S} S-\frac{1}{3 !} S^{2} \dot{S}+\cdots\right)\left(I+S+\frac{1}{2 !} S^{2}+\frac{1}{3 !} S^{3}+\cdots\right) \\
& =\left(-\dot{S}+\frac{1}{2 !} S \dot{S}-\frac{1}{2 !} \dot{S} S-\frac{1}{3 !} \dot{S} S^{2}+\frac{1}{3} S \dot{S} S-\frac{1}{3 !} S^{2} \dot{S}+\cdots\right) \\
& =-[\dot{S}, S]^{(0)}-\frac{1}{2 !}[\dot{S}, S]^{(1)}-\frac{1}{3 !}[\dot{S}, S]^{(2)} \cdots=-\sum_{j=0}^{\infty} \frac{1}{(j+1) !}[\dot{S}, S]^{(j)} .
\end{aligned}
$$


The transformed Hamiltonian then equals

$$
\tilde{\mathcal{H}}=\sum_{j=0}^{\infty} \frac{1}{j !}[\mathcal{H}, S]^{(j)}-i \hbar \sum_{j=0}^{\infty} \frac{1}{(j+1) !}[\dot{S}, S]^{(j)}
$$

with $[\mathcal{H}, S]^{(n+1)}=\left[[\mathcal{H}, S]^{(n)}, S\right]$ and $[\mathcal{H}, S]^{(0)}=\mathcal{H}$. Note that the second term in (A4) is new with respect to time-independent $\mathrm{SW}$, and it is a consequence of the time dependence of the Hamiltonian and therefore that of the matrix $S$. Considering a time-independent Hamiltonian the second term vanishes and we are left with the well-known SW transformation.

We now exploit the block-off-diagonal property of $S$ in order to separate the block-off-diagonal and block-diagonal parts of the transformed Hamiltonian:

$$
\begin{aligned}
& \tilde{\mathcal{H}}_{\text {off-diag }}=\sum_{j=0}^{\infty} \frac{1}{(2 j+1) !}\left[\mathcal{H}_{0}+\mathcal{H}_{1}, S\right]^{(2 j+1)}+\sum_{j=0}^{\infty} \frac{1}{(2 j) !}\left[\mathcal{H}_{2}, S\right]^{(2 j)}-i \hbar \sum_{j=0}^{\infty} \frac{1}{(2 j+1) !}[\dot{S}, S]^{(2 j)}, \\
& \tilde{\mathcal{H}}_{\text {diag }}=\sum_{j=0}^{\infty} \frac{1}{(2 j) !}\left[\mathcal{H}_{0}+\mathcal{H}_{1}, S\right]^{(2 j)}+\sum_{j=0}^{\infty} \frac{1}{(2 j+1) !}\left[\mathcal{H}_{2}, S\right]^{(2 j+1)}-i \hbar \sum_{j=0}^{\infty} \frac{1}{(2 j+2) !}[\dot{S}, S]^{(2 j+1)} .
\end{aligned}
$$

Then, $S$ is determined by solving

$$
\tilde{\mathcal{H}}_{\text {off-diag }}=0 .
$$

The effective (now block-diagonal) Hamiltonian becomes $\tilde{\mathcal{H}}=\tilde{\mathcal{H}}_{\text {diag. }}$. Note that $\tilde{\mathcal{H}}$ as well as the term "effective Hamiltonian" is also used to describe the block of $\tilde{\mathcal{H}}$ corresponding to the relevant subspace.

So far no approximation has been made; now we make use of the smallness of the perturbation. Following the approach of time-independent SW perturbation theory, we aim at solving Eq. (A7) via expanding $S$ as a power series in the perturbation,

$$
S=S_{1}+S_{2}+S_{3}+\cdots,
$$

where $S_{j}$ represents an operator of $j$ th order in the perturbation. Recall that in TDSW, $S$ is time-dependent, and its time derivative appears in its defining equation (A7) as well as in the effective Hamiltonian (A6). Therefore, to separate the terms of different order in perturbing parameter in Eq. (A7), it is necessary to make an a priori assumption on the frequency scale characterizing the magnitude of $\dot{S}_{j}$. As the drive frequency is $\omega$, expectedly the frequency characterizing the time evolution of all $S_{j}$ 's will be $\sim \omega$, hence we assume $\dot{S}_{j} \sim \omega S_{j}$. In the EDSR problem defined in Sec. IV A, the relevant subspace is the subspace of the ground-state orbital spanned by $\left|0_{\uparrow}\right\rangle$ and $\left|0_{\downarrow}\right\rangle$. Furthermore, the energy scales of the drive frequency, drive strength, Zeeman splitting, and spin-orbit coupling are much lower than the splitting between the oscillator levels $\sim \omega_{0}$, and all of them are treated as perturbation. This implies that $\dot{S}_{j}$ is of the order of $(j+1)$ in perturbation.

Obviously, after solving Eq. (A7) with this assumption, we need to check whether the obtained $S_{j}$ functions are consistent with our assumption above.

From the order-by-order expansion of Eq. (A7), we obtain the following hierarchy of simple algebraic equations for the $S_{j}$ matrices:

$$
\begin{aligned}
& {\left[\mathcal{H}_{0}, S_{1}\right]=-\mathcal{H}_{2},} \\
& {\left[\mathcal{H}_{0}, S_{2}\right]=-\left[\mathcal{H}_{1}, S_{1}\right]+i \hbar \dot{S}_{1},} \\
& {\left[\mathcal{H}_{0}, S_{3}\right]=-\left[\mathcal{H}_{1}, S_{2}\right]-\frac{1}{3}\left[\mathcal{H}_{2}, S_{1}\right]^{(2)}+i \hbar \dot{S}_{2},}
\end{aligned}
$$

$$
\begin{aligned}
{\left[\mathcal{H}_{0}, S_{4}\right]=} & -\left[\mathcal{H}_{1}, S_{3}\right]-\frac{1}{3}\left[\left[\mathcal{H}_{2}, S_{1}\right], S_{2}\right] \\
& -\frac{1}{3}\left[\left[\mathcal{H}_{2}, S_{2}\right], S_{1}\right]+i \hbar \dot{S}_{3},
\end{aligned}
$$

Once the first equation (A9a) is solved for $S_{1}(t)$, the solution can be inserted to (A9a) which then forms an algebraic equation for $S_{2}(t)$, etc. Note that since we work in the eigenbasis of $\mathcal{H}_{0}$, the above procedure simplifies to subsequently solving single-variable linear equations, which is a trivial analytical task, well suited for symbolic computation.

After obtaining the $S_{j}$ matrices and inserting them into Eq. (A6), we have an order-by-order expansion $\tilde{\mathcal{H}}=\tilde{\mathcal{H}}_{\text {diag }}=$ $\sum_{j=0}^{\infty} \tilde{\mathcal{H}}^{(n)}$, where

$$
\begin{aligned}
\tilde{\mathcal{H}}^{(0)}= & \mathcal{H}_{0}, \\
\tilde{\mathcal{H}}^{(1)}= & \mathcal{H}_{1}, \\
\tilde{\mathcal{H}}^{(2)}= & {\left[\mathcal{H}_{2}, S_{1}\right]+\frac{1}{2}\left[\mathcal{H}_{0}, S_{1}\right]^{(2)} } \\
\tilde{\mathcal{H}}^{(3)}= & {\left[\mathcal{H}_{2}, S_{2}\right]+\frac{1}{2}\left[\mathcal{H}_{1}, S_{1}\right]^{(2)}+\frac{1}{2}\left[\left[\mathcal{H}_{0}, S_{1}\right], S_{2}\right] } \\
& +\frac{1}{2}\left[\left[\mathcal{H}_{0}, S_{2}\right], S_{1}\right]-i \hbar \frac{1}{2}\left[\dot{S}_{1}, S_{1}\right]
\end{aligned}
$$

With the use of Eqs. (A9) we can further simplify Eqs. (A10):

$$
\begin{aligned}
\tilde{\mathcal{H}}^{(0)} & =\mathcal{H}_{0}, \\
\tilde{\mathcal{H}}^{(1)} & =\mathcal{H}_{1}, \\
\tilde{\mathcal{H}}^{(2)} & =\frac{1}{2 !}\left[\mathcal{H}_{2}, S_{1}\right], \\
\tilde{\mathcal{H}}^{(3)} & =\frac{1}{2 !}\left[\mathcal{H}_{2}, S_{2}\right], \\
\tilde{\mathcal{H}}^{(4)} & =\frac{1}{2 !}\left[\mathcal{H}_{2}, S_{3}\right]-\frac{1}{4 !}\left[\mathcal{H}_{2}, S_{1}\right]^{(3)}, \\
\tilde{\mathcal{H}}^{(5)} & =\frac{1}{2 !}\left[\mathcal{H}_{2}, S_{4}\right]-\frac{1}{4 !}\left[\left[\left[\mathcal{H}_{2}, S_{1}\right], S_{1}\right], S_{2}\right]
\end{aligned}
$$




$$
-\frac{1}{4 !}\left[\left[\left[\mathcal{H}_{2}, S_{1}\right], S_{2}\right], S_{1}\right]-\frac{1}{4 !}\left[\left[\left[\mathcal{H}_{2}, S_{2}\right], S_{1}\right], S_{1}\right]
$$

Finally, we need to check the consistency of our assumption for the time evolution of $S_{j}$ with the actual solution we obtained for $S_{j}$ using that assumption. From (A9a), $S_{1}$ inherits harmonic time dependence from $\mathcal{H}_{2}$ with frequency $\omega$. This implies that the time derivative is $\dot{S}_{1} \sim \omega S_{1}$, as assumed. From Eq. (A9b), the matrix $S_{2}$ might contain frequency components at $\omega$, as well as at zero frequency and $2 \omega$ (if $\mathcal{H}_{1}$ is time-dependent with frequency $\omega$ ); nevertheless, the $\dot{S}_{2} \sim \omega S_{2}$ relation still holds, etc.

In conclusion, TDSW allows for obtaining an effective time-dependent Hamiltonian for the relevant subspace. The procedure is to evaluate the transformation matrices $S_{j}$ up to the desired order via solving Eq. (A9), and substituting the resulting $S_{j}$ matrices into Eq. (A11).

\section{APPENDIX B: RABI FREQUENCY OF THE FUNDAMENTAL RESONANCE: RELATION TO THE RESULTS OF REFERENCE [5]}

EDSR in a QD in a two-dimensional electron gas due to Rashba and Dresselhaus spin-orbit interactions has been described by Golovach, Borhani, and Loss (GBL) in Ref. [5]. Therein, the Rabi frequency of the fundamental resonance as a function of system parameters (magnetic field strength, magnetic field direction, spin-orbit interaction strengths, and ac electric field amplitude and direction) has been calculated. Even though the dimensionality and the spin-orbit Hamiltonian in the model of GBL differ from our model, the calculated Rabi frequencies can be compared after a special case of the model of GBL has been reduced to one dimension. Here we show that after this dimension reduction our result for the fundamental Rabi frequency equals that of GBL.
In the model of GBL, the 2DEG lies in the $x-y$ plane. We consider the special case when the confinement potential is parabolic and has a cylindrical symmetry, the Dresselhaus coupling vanishes, $\beta=0$, the $B$ field is in the $y-z$ plane, and the $E$ field is along the $x$ axis. Furthermore, we project the Hamiltonian on the $y$-ground-state orbital of the harmonic oscillator, yielding

$$
\begin{aligned}
H_{\mathrm{GBL}}= & \frac{p_{x}^{2}}{2 m}+\frac{1}{2} m \omega_{0}^{2} x^{2}+\alpha p_{x} \sigma_{y}+\frac{1}{2} g^{*} \mu_{B} \mathbf{B} \cdot \boldsymbol{\sigma} \\
& +e E_{\mathrm{ac}} x \sin \omega t .
\end{aligned}
$$

For simplicity, we focus on the special case $\mathbf{B}=(0,0, B)$ from now on. Then, the Hamiltonian in Eq. (B1) is equivalent to our Hamiltonian $\mathcal{H}$ at $\theta=0$.

To deduce the Rabi frequency calculated by GBL for the special case above, we start from their Eqs. (13) and (14), where they provide the time-dependent part of the effective qubit Hamiltonian as

$$
H_{\mathrm{GBL}}=\frac{1}{2} \mathbf{h}(t) \cdot \boldsymbol{\sigma},
$$

where

$$
\mathbf{h}(t)=2 \mu_{B} \mathbf{B} \times \boldsymbol{\Omega}(t) .
$$

A straightforward calculation shows that

$$
\begin{aligned}
\frac{1}{2} \mathbf{h}(t) & =\frac{\alpha e E_{\mathrm{ac}} g^{*} \mu_{B} B}{\hbar \omega_{0}^{2}} \sin (\omega t) \mathbf{e}_{x} \\
& =2 \frac{\tilde{\alpha} \tilde{E}_{\mathrm{ac}} \tilde{B}}{\hbar^{2} \omega_{0}^{2}} \sin (\omega t) \mathbf{e}_{x} .
\end{aligned}
$$

Note that this effective ac magnetic field is perpendicular to the static magnetic field, which is applied in the $z$ direction. The Rabi frequency due to this ac magnetic field at the fundamental resonance frequency reads

$$
\Omega_{\mathrm{res}, \mathrm{GBL}}^{(1)}=2 \frac{\tilde{\alpha} \tilde{E}_{\mathrm{ac}} \tilde{B}}{\hbar^{2} \omega_{0}^{2}},
$$

which is identical to our result in Eq. (8), if the latter is evaluated at $\theta=0$ and terms above third order are dropped.
[1] F. Bloch and A. Siegert, Phys. Rev. 57, 522 (1940).

[2] J. H. Shirley, Phys. Rev. 138, B979 (1965).

[3] F. H. L. Koppens, C. Buizert, K. J. Tielrooij, I. T. Vink, K. C. Nowack, T. Meunier, L. P. Kouwenhoven, and L. M. K. Vandersypen, Nature (London) 442, 766 (2006).

[4] M. Veldhorst, J. C. C. Hwang, C. H. Yang, A. W. Leenstra, B. de Ronde, J. P. Dehollain, J. T. Muhonen, F. E. Hudson, K. M. Itoh, A. Morello, and A. S. Dzurak, Nat. Nanotechnol. 9, 981 (2014).

[5] V. N. Golovach, M. Borhani, and D. Loss, Phys. Rev. B 74, 165319 (2006).

[6] C. Flindt, A. S. Sørensen, and K. Flensberg, Phys. Rev. Lett. 97, 240501 (2006).

[7] Y. Tokura, W. G. van der Wiel, T. Obata, and S. Tarucha, Phys. Rev. Lett. 96, 047202 (2006).

[8] E. I. Rashba, Phys. Rev. B 78, 195302 (2008).

[9] J. D. Walls, Phys. Rev. B 76, 195307 (2007).
[10] D. V. Khomitsky, L. V. Gulyaev, and E. Y. Sherman, Phys. Rev. B 85, 125312 (2012).

[11] R. Li, J. Q. You, C. P. Sun, and F. Nori, Phys. Rev. Lett. 111, 086805 (2013).

[12] Y. Kato, R. C. Myers, D. C. Driscoll, A. C. Gossard, J. Levy, and D. D. Awschalom, Science 299, 1201 (2003).

[13] K. C. Nowack, F. H. L. Koppens, Y. V. Nazarov, and L. M. K. Vandersypen, Science 318, 1430 (2007).

[14] M. Pioro-Ladriere, T. Obata, Y. Tokura, Y.-S. Shin, T. Kubo, K. Yoshida, T. Taniyama, and S. Tarucha, Nat. Phys. 4, 776 (2008).

[15] E. A. Laird, C. Barthel, E. I. Rashba, C. M. Marcus, M. P. Hanson, and A. C. Gossard, Phys. Rev. Lett. 99, 246601 (2007).

[16] S. Nadj-Perge, S. M. Frolov, E. P. A. M. Bakkers, and L. P. Kouwenhoven, Nature (London) 468, 1084 (2010). 
[17] S. Nadj-Perge, V. S. Pribiag, J. W. G. van den Berg, K. Zuo, S. R. Plissard, E. P. A. M. Bakkers, S. M. Frolov, and L. P. Kouwenhoven, Phys. Rev. Lett. 108, 166801 (2012).

[18] F. Pei, E. A. Laird, G. A. Steele, and L. P. Kouwenhoven, Nat. Nanotechnol. 7, 630 (2012).

[19] V. S. Pribiag, S. Nadj-Perge, S. M. Frolov, J. W. G. van den Berg, I. van Weperen, S. R. Plissard, E. P. A. M. Bakkers, and L. P. Kouwenhoven, Nat. Nanotechnol. 8, 170 (2013).

[20] E. A. Laird, F. Pei, and L. P. Kouwenhoven, Nat. Nanotechnol. 8, 565 (2013).

[21] E. Kawakami, P. Scarlino, D. R. Ward, F. R. Braakman, D. E. Savage, M. G. Lagally, M. Friesen, S. N. Coppersmith, M. A. Eriksson, and L. M. K. Vandersypen, Nat. Nanotechnol. 9, 666 (2014).

[22] P. V. Klimov, A. L. Falk, B. B. Buckley, and D. D. Awschalom, Phys. Rev. Lett. 112, 087601 (2014).

[23] F. Forster, M. Mühlbacher, D. Schuh, W. Wegscheider, and S. Ludwig, Phys. Rev. B 91, 195417 (2015).

[24] E. A. Laird, C. Barthel, E. I. Rashba, C. M. Marcus, M. P. Hanson, and A. C. Gossard, Semicond. Sci. Technol. 24, 064004 (2009).

[25] M. D. Schroer, K. D. Petersson, M. Jung, and J. R. Petta, Phys. Rev. Lett. 107, 176811 (2011).

[26] J. Stehlik, M. D. Schroer, M. Z. Maialle, M. H. Degani, and J. R. Petta, Phys. Rev. Lett. 112, 227601 (2014).

[27] A. De, C. E. Pryor, and M. E. Flatté, Phys. Rev. Lett. 102, 017603 (2009).

[28] J. Pingenot, C. E. Pryor, and M. E. Flatté, Phys. Rev. B 84, 195403 (2011).

[29] E. I. Rashba, Phys. Rev. B 84, 241305 (2011).
[30] M. P. Nowak, B. Szafran, and F. M. Peeters, Phys. Rev. B 86, 125428 (2012).

[31] E. N. Osika, B. Szafran, and M. P. Nowak, Phys. Rev. B 88, 165302 (2013).

[32] G. Széchenyi and A. Pályi, Phys. Rev. B 89, 115409 (2014).

[33] E. N. Osika, A. Mreńca, and B. Szafran, Phys. Rev. B 90, 125302 (2014).

[34] J. Danon and M. S. Rudner, Phys. Rev. Lett. 113, 247002 (2014).

[35] M. Trif, V. N. Golovach, and D. Loss, Phys. Rev. B 77, 045434 (2008).

[36] T. Dittrich, Quantum Transport and Dissipation (Wiley-VCH Verlag GmbH, Weinheim, 1998).

[37] P. K. Aravind and J. O. Hirschfelder, J. Phys. Chem. 88, 4788 (1984).

[38] I. Gromov and A. Schweiger, J. Magn. Res. 146, 110 (2000).

[39] J. R. Schrieffer and P. A. Wolff, Phys. Rev. 149, 491 (1966).

[40] R. Winkler, Spin-orbit Coupling Effects in Two-Dimensional Electron and Hole Systems, Springer Tracts in Modern Physics, Vol. 191 (Springer-Verlag, Berlin, Heidelberg, 2003).

[41] C. Wei, A. S. M. Windsor, and N. B. Manson, J. Phys. B: At. Mol. Opt. Phys. 30, 4877 (1997).

[42] L. S. Levitov and E. I. Rashba, Phys. Rev. B 67, 115324 (2003).

[43] P. Scarlino, E. Kawakami, D. R. Ward, D. E. Savage, M. G. Lagally, M. Friesen, S. N. Coppersmith, M. A. Eriksson, and L. M. K. Vandersypen, arXiv:1504.06436.

[44] F. Schwabl, Advanced Quantum Mechanics (Springer-Verlag, Berlin, Heidelberg, 2008), Chap. 9.

[45] A. Kaminski, Y. V. Nazarov, and L. I. Glazman, Phys. Rev. B 62, 8154 (2000).

[46] Y. Goldin and Y. Avishai, Phys. Rev. B 61, 16750 (2000). 